Pacific Journal of Mathematics

CARATHÉODORY CONVEX INTEGRAND OPERATORS AND

NiKOLAOS S. PAPAGEORGIOU 


\title{
CARATHEODORY CONVEX INTEGRAND OPERATORS AND PROBABILITY THEORY
}

\author{
Nikolaos S. Papageorgiou
}

In two recent papers, the author studied extensions of several concepts of nonsmooth analysis to vector valued operators. The purpose of the present work is to further continue this effort and to study, from a probabilistic viewpoint, several properties of convex operators. In particular, we will examine how various basic concepts of vectorial nonsmooth analysis associated with an integrand $f(\omega, x)$ are related to those of the integral operator $F(x)=\int_{\Omega} f(\omega, x) d \mu(\omega)$ where the vector valued integral is defined in the sense of Bochner. Also we introduce a conditional expectation for such integrands, study several of its properties, see how it is affected by various operations of nonsmooth analysis, and derive a vector valued martingale convergence theorem.

1. For real valued functions, the most important contributions on this subject were made by Rockafellar $[17,18,19,20]$, who introduced the notion of the normal integrand. Caratheodory integrands form a subset in the family of normal integrands. Following Rockafellar, interesting results were also obtained by Bismut [3] and Hiriart-Urruty [10]. Our work generalizes several of their results to a vector valued context.

In our presentation, we will use parts of the general theory of ordered topological vector spaces. For the necessary background on this topic, we refer the reader to the books by Peressini [16] and Schaefer [23].

2. Preliminaries. We start by recalling some basic facts from the theory of measurable multifunctions. For more details the reader can refer to Castaing-Valadier [4], Himmelberg [9] or Rockafellar [20].

Let $F: \Omega \rightarrow 2^{X}$ be a multivalued function (multifunction) from a space to the family of subsets of a space $X$. We introduce the set

$$
\operatorname{Gr} F=\{(\omega, x) \in \Omega \times X: x \in F(\omega)\}
$$

which we call the graph of $F$. Also for $V \subseteq X$, we define $F^{-}(V)=\{\omega \in \Omega$ : $F(\omega) \cap V \neq \varnothing\}$. If $X$ is a topological space, by $P_{f}(X)$ we denote the nonempty and closed subsets of $X$.

The next theorem summarizes the interrelations of the different notions of measurability of a multifunction that exist in the literature. 
TheOREM 2.1. Let $(\Omega, \Sigma)$ be a measurable space and $X$ a separable metric space. Let $F: \Omega \rightarrow P_{f}(X)$ be a multifunction. Consider the following statements.

(1) $F^{-}(B) \in \Sigma$ for every $B \in B(X)=$ the Borel $\sigma$-field of $X$.

(2) $F^{-}(C) \in \Sigma$ for every $C$ closed subset of $X$.

(3) $F^{-}(U) \in \Sigma$ for every $U$ open subset of $X$.

(4) $\omega \rightarrow d(x, F(\omega))$ is a measurable function for every $x \in X$.

(5) There exists a sequence of measurable selectors $\left\{f_{n}\right\}_{n \geq 1}$ of $F(\cdot)$ s.t. $\operatorname{cl}\left\{f_{n}(\omega)\right\}_{n \geq 1}=F(\omega)$ (Castaing representation).

(6) $\mathrm{Gr} F \in \Sigma \times B(X)$.

Then we have the following relations among them:

(i) (1) $\rightarrow$ (2) $\rightarrow$ (3) $\leftrightarrow$ (4) $\rightarrow$ (6).

(ii) If $X$ is Polish (i.e., is, in addition, complete) then (3) $\leftrightarrow$ (5).

(iii) If $X$ is Polish and there is a complete $\sigma$-finite measure on $\Omega$, then (1) to (6) are all equivalent.

Following Himmelberg [9], we say that an $F(\cdot)$ satisfying (1) (resp. (2), (3)) is Borel (resp. strongly, weakly) measurable.

Let $(\Omega, \Sigma, \mu)$ be a measure space and $X$ a separable Banach space. For a multifunction $F: \Omega \rightarrow P_{f}(X)$ we define the set

$$
S_{F}^{1}=\left\{f(\cdot) \in L_{X}^{1}(\Omega): f(\omega) \in F(\omega) \mu \text {-a.e. }\right\},
$$

i.e. $S_{F}^{1}$ contains all integrable selectors of $F(\cdot)$.

It is easy to see that $S_{F}^{1}$ is a closed subset of $L_{X}^{1}(\Omega)$ and it is convex if and only if $F(\omega)$ is $\mu$-a.e. convex. Furthermore, this set may very well be empty.

Using $S_{F}^{1}$ we can now define an integral for the multifunction $F(\cdot)$. This integral was first introduced by Aumann [2] for $X=R^{n}$.

$$
\int_{\Omega} F(\omega) d \mu(\omega)=\left\{\int_{\Omega} f(\omega) d \mu(\omega): f \in S_{F}^{1}\right\} .
$$

As we already mentioned, the vector integrals should be interpreted as Bochner integrals. Clearly $\int_{\Omega} F(\omega) d \mu(\omega)=\varnothing$ if $S_{F}^{1}=\varnothing$.

We say that a measurable multifunction $F: \Omega \rightarrow P_{f}(X)$ is integrably bounded if there is a $\phi(\cdot) \in L^{1}(\Omega)$ s.t.

$$
|F(\omega)|=\sup _{x \in F(\omega)}\|x\| \leq \phi(\omega) \quad \mu \text {-a.e. }
$$

For an integrably bounded multifunction $F(\cdot)$ as above, using the Kuratowski-Ryll Nardzewski measurable selection theorem (see [9] or [20]), we see that $S_{F}^{1} \neq \varnothing$, and $\int_{\Omega} F(\omega) d \mu(\omega) \neq \varnothing$. 
In [8], Hiai and Umegaki proved that if $F: \Omega \rightarrow P_{f}(X)$ is integrably bounded, then there is a unique $E^{\Sigma_{0}} F: \Omega \rightarrow P_{f}(X) \Sigma_{0}$-measurable s.t.

$$
S_{E^{\Sigma_{0 F}}}^{1}\left(\Sigma_{0}\right)=\operatorname{cl}\left\{E^{\Sigma_{0}} f: f \in S_{F}^{1}\right\},
$$

the closure being taken in $L_{X}^{1}(\Omega)$. Also for every $A \in \Sigma_{0}$, we have

$$
\operatorname{cl} \int_{A}^{\left(\Sigma_{0}\right)} E^{\Sigma_{0}} F(\omega) d \mu(\omega)=\operatorname{cl} \int_{A} F(\omega) d \mu(\omega),
$$

where

$$
\int_{A}^{\left(\Sigma_{0}\right)} E^{\Sigma_{0}} F(\omega) d \mu(\omega)=\left\{\int_{\Omega} g(\omega) d \mu(\omega), g \in S_{E^{\Sigma_{0}}}^{1}\left(\Sigma_{0}\right)\right\} .
$$

Finally, we very briefly recall some useful facts about the Hausdorff metric. If $A, B \in P_{f}(X)$, their Hausdorff distance is defined by

$$
h(A, B)=\max \left\{\sup _{a \in A} d(a, B), \sup _{b \in B} d(b, A)\right\}
$$

where $d(\cdot, \cdot)$ denotes the distance function. It is easy to check that $h(\cdot, \cdot)$ is a metric on $P_{f}(X)$ which is a complete metric space (the same is true for $P_{f c}(X)$, which is the family of all nonempty, closed convex, subsets of $X$ ).

If $A, B$ are nonempty, bounded, closed convex subsets of $X$, their Hausdorff distance can also be given through Hörmander's formula (see [4]).

$$
h(A, B)=\sup _{\left\|x^{*}\right\| \leq 1}\left|\sigma_{A}\left(x^{*}\right)-\sigma_{B}\left(x^{*}\right)\right| .
$$

Here $\sigma_{A}(\cdot)\left(\sigma_{B}(\cdot)\right)$ is the support functional of $A(B)$.

3. Convex operators. Throughout the rest of this paper $(\Omega, \Sigma, \mu)$ will be a complete probability space, $X$ a separable Banach space and $Y$ a separable w-sequentially complete Banach lattice which is order complete (see [16]).

An operator $f: X \rightarrow Y$ is said to be convex if and only if for any $x_{1}$, $x_{2} \in X$ and all $\lambda \in[0,1]$,

$$
\lambda f\left(x_{1}\right)+(1-\lambda) f\left(x_{2}\right)-f\left(\lambda x_{1}+(1-\lambda) x_{2}\right) \in Y_{+},
$$

and, in a more suggestive notation,

$$
f\left(\lambda x_{1}+(1-\lambda) x_{2}\right) \leq \lambda f\left(x_{1}\right)+(1-\lambda) f\left(x_{2}\right) .
$$

We mainly consider Caratheodory integrand operators $f(\omega, x)$, although in the last section we obtain results for more general integrand operators. 
For now, let $f: \Omega \times X \rightarrow Y$ be an integrand operator with the following properties.

(1) $f(\omega, \cdot)$ is continuous, convex for $\mu$-almost all $\omega \in \Omega$.

(2) $f(\cdot, x)$ is measurable for all $x \in X$.

(3) $\|f(\omega, x)\| \leq h(\omega) \mu$-a.e. for some $h(\cdot) \in L^{1}(\Omega)$ and for all $x \in X$.

Note that in (2), we don't specify the kind of measurability of $f(\cdot, x)$, since because of the separability of $Y$, strong (Bochner), weak (Pettis), and Borel measurability all coincide (see [6]).

An operator $f(\cdot, \cdot)$ satisfying (1), (2) and (3) is called a Caratheodory, convex, $L_{Y}^{1}(\Omega)$-integrand operator. Consider the Lebesgue-Bochner spaces $L_{X}^{p}(\Omega)(1 \leq p \leq \infty)$. Then on those spaces, we define the following integral operator (usually known as the Nemitsky or superposition operator):

$$
F(u)=\int_{\Omega} f(\omega, u(\omega)) d \mu(\omega), \quad \text { where } u(\cdot) \in L_{X}^{p}(\Omega)(1 \leq p \leq \infty) .
$$

It is easy to check that $F(\cdot)$ is a norm continuous convex operator on $L_{X}^{p}(\Omega)$.

We start with an easy, but useful, Lemma.

LEMMA $\alpha$. If $f_{1}(\cdot), f_{2}(\cdot) \in L_{Y}^{1}(\Omega)$ and $f_{1}(\omega) \leq f_{2}(\omega) \mu$-a.e., then

$$
\int_{\Omega} f_{1}(\omega) d \mu(\omega) \leq \int_{\Omega} f_{2}(\omega) d \mu(\omega) .
$$

Proof. Let $y^{*} \in Y_{+}^{*}$. Then $\left(y^{*}, f_{2}(\omega)-f_{1}(\omega)\right) \geq 0 \mu$-a.e. So $\int_{\Omega}\left(y^{*}, f_{2}(\omega)-f_{1}(\omega)\right) d \mu(\omega) \geq 0$. But

$$
\int_{\Omega}\left(y^{*}, f_{2}(\omega)-f_{1}(\omega)\right) d \mu(\omega)=\left(y^{*}, \int_{\Omega}\left(f_{2}(\omega)-f_{1}(\omega)\right) d \mu(\omega)\right) .
$$

Hence for all $y^{*} \in Y_{+}^{*},\left(y^{*}, \int_{\Omega}\left(f_{2}(\omega)-f_{1}(\omega)\right) d \mu(\omega)\right) \geq 0$, which implies

$$
\int_{\Omega} f_{1}(\omega) d \mu(\omega) \leq \int_{\Omega} f_{2}(\omega) d \mu(\omega) .
$$

Another useful preliminary result is

Lemma $\beta$. A Caratheodory operator (not necessarily convex) is superpositionally measurable, i.e. for every $u: \Omega \rightarrow$ X measurable, $\omega \rightarrow f(\omega, u(\omega))$ is also measurable.

Proof. Since $u: \Omega \rightarrow X$ is measurable, we know there exist simple functions $s_{n}(\omega)=\sum_{k=1}^{m} x_{n k} \chi_{A_{n k}}(\omega)$ s.t. $s_{n}(\omega) \stackrel{\mathrm{s}}{\rightarrow} u(\omega) \mu$ a.e. Then from the 
continuity of $f(\omega, \cdot)$, we have $f\left(\omega, s_{n}(\omega)\right) \rightarrow f(\omega, u(\omega)) \mu$-a.e. Let $B \in$ $B(X)$ and consider the set

$$
U_{n}=\left\{\omega \in \Omega: f\left(\omega, s_{n}(\omega)\right) \in B\right\} .
$$

Clearly, $U_{n}=\bigcup_{k=1}^{m} f_{x_{n k}}^{-1}(U) \cap A_{n k}$ where $f_{x_{n k}}(\omega)=f\left(\omega, x_{n k}\right)$. Hence $U_{n} \in$ $\Sigma$. Therefore for $n \geq 1, \omega \rightarrow f\left(\omega, s_{n}(\omega)\right)$ is measurable, so their limit $\omega \rightarrow f(\omega, u(\omega))$ is also measurable too.

Since $F: L_{X}^{p}(\Omega) \rightarrow Y$ is a convex operator, we can define its o-directional derivative by

$$
F^{\prime}(u ; h)=\inf _{\lambda>0} \frac{F(u+\lambda h)-F(u)}{\lambda}=\operatorname{oim}_{\lambda \downarrow 0} \frac{F(u+\lambda h)-F(u)}{\lambda}
$$

for $h \in L_{X}^{p}(\Omega)$ (see [12]). Here o-lim denotes the order limit.

TheOREM 3.1. For all $u(\cdot), h(\cdot) \in L_{x}^{p}(\Omega)$, we have

$$
F^{\prime}(u ; h)=\int_{\Omega} f^{\prime}(\omega, u(\omega) ; h(\omega)) d \mu(\omega) .
$$

Proof. We have the check that the integral in the conclusion of the theorem is well defined. From Lemma 5 of [24],

$$
f^{\prime}(\omega, u(\omega) ; h(\omega))=\underset{\substack{\mathrm{w} \\ \lambda \downarrow 0}}{ } \frac{f(\omega, u(\omega)+\lambda h(\omega))-f(\omega, u(\omega))}{\lambda} .
$$

From Lemma $\beta$ we know that the above quotients are measurable. So $f^{\prime}(\omega, u(\omega) ; h(\omega))$ being the weak limit of measurable operators is itself measurable. Hence it remains to show that $\left\|f^{\prime}(\omega, u(\omega) ; h(\omega))\right\| \in L^{1}(\Omega)$. From the definition of the o-directional derivative, we have

$$
\begin{aligned}
& f(\omega, u(\omega))-f(\omega, u(\omega)-h(\omega)) \\
& \leq f^{\prime}(\omega, u(\omega) ; h(\omega)) \leq f(\omega, u(\omega)+h(\omega))-f(\omega, u(\omega)) \\
& \rightarrow\left|f^{\prime}(\omega, u(\omega) ; h(\omega))\right| \leq[f(\omega, u(\omega)+h(\omega))-f(\omega, u(\omega))] \\
& \vee[f(\omega, u(\omega)-h(\omega))-f(\omega, u(\omega))] \\
&= \frac{1}{2}[f(\omega, u(\omega)+h(\omega))+f(\omega, u(\omega)-h(\omega)) \\
& \quad+|f(\omega, u(\omega)+h(\omega))-f(\omega, u(\omega)-h(\omega))|+|f(\omega, u(\omega))| \\
& \leq|f(\omega, u(\omega)+h(\omega))|+\mid f(\omega, u(\omega)-h(\omega)|+| f(\omega, u(\omega)) \mid .
\end{aligned}
$$

From the inequality, since $Y$ is a Banach lattice, we get

$$
\begin{aligned}
\left\|f^{\prime}(\omega, u(\omega) ; h(\omega))\right\| \leq & \|f(\omega, u(\omega)+h(\omega))\|+\|f(\omega, u(\omega)-h(\omega))\| \\
& +\|f(\omega, u(\omega))\| .
\end{aligned}
$$


Hence $\left\|f^{\prime}(\omega, u(\omega) ; h(\omega))\right\| \in L^{1}(\Omega)$, which proves that it is Bochner integrable. Next, for any $h(\cdot) \in L_{X}^{p}(\Omega)$, by definition, we have

$$
\begin{aligned}
& F^{\prime}(u ; h)=\underset{\lambda_{n} \downarrow 0}{\mathrm{o}-\lim _{0}} \frac{F\left(u+\lambda_{n} h\right)-F(u)}{\lambda_{n}}=\underset{\lambda_{n} \downarrow 0}{\mathrm{w}-\lim _{0}} \frac{F\left(u+\lambda_{n} h\right)-F(u)}{\lambda_{n}} \\
& =\mathrm{w}_{\lambda_{n} \downarrow 0} \lim _{\Omega} \frac{f\left(\omega, u(\omega)+\lambda_{n} h(\omega)\right)-f(\omega, u(\omega))}{\lambda_{n}} d \mu(\omega) .
\end{aligned}
$$

So for every $y^{*} \in Y^{*}$,

$$
\begin{aligned}
& \left(y^{*}, F^{\prime}(u ; h)\right) \\
& \quad=\lim _{n \rightarrow \infty}\left(y^{*}, \int_{\Omega} \frac{f\left(\omega, u(\omega)+\lambda_{n} h(\omega)\right)-f(\omega, u(\omega))}{\lambda_{n}} d \mu(\omega)\right) \\
& \quad=\lim _{n \rightarrow \infty} \int_{\Omega}\left(y^{*}, \frac{f\left(\omega, u(\omega)+\operatorname{lam}_{n} h(\omega)\right)-f(\omega, u(\omega))}{\lambda_{n}}\right) d \mu(\omega),
\end{aligned}
$$

and from the Lebesgue dominated convergence theorem,

$$
\begin{aligned}
& \left(y^{*}, F^{\prime}(u, h)\right) \\
& \left.\quad=\int_{\Omega} \lim _{n \rightarrow \infty}\left(y^{*}, \frac{f\left(\omega, u(\omega)+\lambda_{n} h(\omega)\right)-f(\omega, u(\omega))}{\lambda_{n}}\right) d \mu d \omega\right) \\
& \quad=\int_{\Omega}\left(y^{*}, f^{\prime}(\omega, u(\omega) ; h(\omega))\right) d \mu(\omega) \\
& \quad=\left(y^{*}, \int_{\Omega} f^{\prime}(\omega, u(\omega) ; h(\omega)) d \mu(\omega)\right) .
\end{aligned}
$$

Since this is true for any $y^{*} \in Y^{*}$, we finally conclude that

$$
F^{\prime}(u, h)=\int_{\Omega} f^{\prime}(\omega, u(\omega) ; h(\omega)) d \mu(\omega) .
$$

Because $f: L_{X}^{p}(\Omega) \rightarrow Y$ is a convex operator, we can define its subdifferential in the sense of convex analysis (see [12, 24]):

$$
\begin{aligned}
\partial F(u)=\left\{A \in \mathscr{L}\left(L_{X}^{p}(\Omega), Y\right): A\left(u^{\prime}\right)-A(u) \leq\right. & F\left(u^{\prime}\right)-F(u) \\
& \text { for all } \left.u^{\prime}(\cdot) \in L_{X}^{p}(\Omega)\right\} .
\end{aligned}
$$

Using results of Valadier [24], we have the following complete characterization of the above, generally multivalued, operator $\partial F(\cdot)$.

THEOREM 3.2. For all $u(\cdot) \in L_{X}^{p}(\Omega), \partial f(u) \neq \varnothing$ is convex and equicontinuous in $\left(L_{X}^{p}(\Omega), Y\right)$ and we have the formula

$$
F^{\prime}(u ; h)=\max \{A(h): A \in \partial F(u)\}, \quad h(\cdot) \in L_{X}^{p}(\Omega) .
$$


Furthermore, if the order intervals of $Y$ are w-compact then $\partial F(u)$ is compact for the weak operator topology on $\mathscr{L}\left(L_{X}^{p}(\Omega), Y\right)$.

Proof. This result is an immediate consequence of Theorem 6 and Corollary 7 in Valadier [24].

REMARK. If $Y$ is reflexive then, its order intervals, being closed and bounded (because of the normality of $Y$ ), are w-compact. For more general conditions that imply the w-compactness of the order intervals of a Banach lattice, we refer to Schaefer [23].

Now that we have introduced the subdifferential of $F(\cdot)$, it is natural to proceed and examine how it is related to the subdifferential of the integrand $f(\cdot, \cdot)$. This issue is resolved in the next theorem.

THEOREM 3.3. If $Y$ has the Radon-Nikodym property then for any $u(\cdot) \in L_{X}^{p}(\Omega)$, we have that $A \in \partial F(u)$ if and only if there is $A(\omega, \cdot) \in$ $L_{\mathscr{L}(X, Y)}^{1}(\Omega)$ s.t. $A(\omega) \in \partial f(\omega, u(\omega)) \mu$-a.e. and $A(\cdot)=\int_{\Omega} A(\omega, \cdot) d \mu(\omega)$.

Proof. We know (see [12]) that $A \in \partial f(u)$ if and only if $A(h) \leq$ $F^{\prime}(u ; h)$ for all $h(\cdot) \in L_{X}^{p}(\Omega)$. Also, from Theorem 3.1, we have

$$
F^{\prime}(u ; h)=\int_{\Omega} f^{\prime}(\omega, u(\omega) ; h(\omega)) d \mu(\omega) .
$$

Note that $f^{\prime}(\omega, u(\omega) ; \cdot)$ is sublinear. Then using a result due to Neumann [11] and Saint Pierre [22], we know that for every $d \in X$,

$$
A(d)=\int_{\Omega} A(\omega, d) d \mu(\omega)
$$

with $A(\omega, u(\omega)) \in \partial f(\omega, u(\omega)) \mu$-a.e. and $A(\cdot, d) \in L_{\mathscr{L}(X, Y)}^{1}(\Omega)$. Consider a simple function $s(\omega)=\sum_{k=1}^{n} x_{k} \chi_{A_{k}}(\omega)$. Then we have

$$
\begin{aligned}
A(s) & =A\left(\sum_{k=1}^{n} x_{k} \chi_{A_{k}}\right)=\sum_{k=1}^{n} A\left(x_{k} \chi_{A_{k}}\right) \\
& =\sum_{k=1}^{n} \int_{A_{k}} A\left(\omega, x_{k}\right) d \mu(\omega)=\sum_{k=1}^{n} \int_{\Omega} A\left(\omega, x_{k} \chi_{A_{k}}(\omega)\right) d \mu(\omega) \\
& =\int_{\Omega} \sum_{k=1}^{n} A\left(\omega, x_{k} \chi_{A_{k}}(\omega)\right) d \mu(\omega)=\int_{\Omega} A\left(\omega, \sum_{k=1}^{n} x_{k} \chi_{A_{k}}(\omega)\right) d \mu(\omega) \\
& =\int_{\Omega} A(\omega, s(\omega)) d \mu(\omega)
\end{aligned}
$$


Let $\left\{s_{n}\right\}_{n \geq 1}$ be a sequence of simple functions s.t. $s_{n}(\omega) \stackrel{s}{\rightarrow} h(\omega) \mu$-a.e. and $\int_{\Omega}\left\|s_{n}(\omega)-h(\omega)\right\| d \mu(\omega) \rightarrow 0$ as $n \rightarrow \infty$. As we just saw,

$$
A\left(s_{n}\right)=\int_{\Omega} A\left(\omega, s_{n}(\omega)\right) d \mu(\omega) .
$$

From the continuity of $A(\cdot)$ and $A(\omega, \cdot) \mu$-a.e.,

$$
A\left(s_{n}\right) \stackrel{s}{\rightarrow} A(s) \text { and } A\left(\omega, s_{n}(\omega)\right) \stackrel{s}{\rightarrow} A(\omega, h(\omega)) \quad \mu \text {-a.e. }
$$

Recall that

$$
-f^{\prime}(\omega, u(\omega) ;-h(\omega)) \leq A(\omega, h(\omega)) \leq f^{\prime}(\omega, u(\omega) ; h(\omega)) \quad \mu \text {-a.e. }
$$

so

$$
\begin{aligned}
|A(\omega, h(\omega))| & \leq\left|f^{\prime}(\omega, u(\omega) ; h(\omega))\right| \vee\left|f^{\prime}(\omega, u(\omega) ;-h(\omega))\right| \\
& \leq\left|f^{\prime}(\omega, u(\omega) ; h(\omega))\right|+\left|f^{\prime}(\omega, u(\omega) ;-h(\omega))\right| \quad \mu \text {-a.e. }
\end{aligned}
$$

Since $Y$ is a Banach lattice, we finally have

$$
\|A(\omega, h(\omega))\| \leq\left\|f^{\prime}(\omega, u(\omega) ; h(\omega))\right\|+\left\|f^{\prime}(\omega, u(\omega) ;-h(\omega))\right\| \quad \mu \text {-a.e. }
$$

Employing the Lebesgue dominated convergence theorem for Bochner integrals, we deduce that

$$
\begin{aligned}
\lim _{n \rightarrow \infty} A\left(s_{n}\right) & =A(h)=\lim _{n \rightarrow \infty} \int_{\Omega} A\left(\omega, s_{n}(\omega)\right) d \mu(\omega) \\
& =\int_{\Omega} A(\omega, h(\omega)) d \mu(\omega) .
\end{aligned}
$$

Therefore we conclude that

$$
A \in \partial F(u) \quad \text { iff } \quad A(\omega) \in \partial f(\omega, u(\omega)) \quad \mu \text {-a.e. }
$$

as desired.

For real valued functions, we know from the work of Rockafellar $[19,20]$ that if $f(\cdot, \cdot)$ is a normal complex integrand (e.g. a Caratheodory convex integrand), then the closed and convex valued multifunction $\omega \rightarrow \partial f(\omega, u(\omega)), u(\cdot) \in L_{X}^{p}(\Omega)$, is measurable. But, when $Y$ is infinite dimensional, we are facing a technical problem. $\mathscr{L}(X, Y)$ is not separable even if $X=Y=$ separable Hilbert space (see Problem 83 in Halmos [7]). So Theorem 2.1 is not applicable in this case. However, we can still say something interesting about the measurability of the subdifferential multifunction.

THEOREM 3.4. For all $u(\cdot) \in L_{X}^{p}(\Omega)$, the subdifferential multifunction $f(\omega, u(\omega))$ has a $\Sigma \times B(X)$ measurable graph. 
Proof. We will start by showing the continuity of $f^{\prime}(\omega, u(\omega) ; \cdot)$ on $X$ for every $\omega \in \Omega$. So fix and $\omega \in \Omega$ and recall that for all $d \in X$,

$$
\begin{aligned}
& f(\omega, u(\omega))-f(\omega, u(\omega)-d) \\
& \quad \leq f^{\prime}(\omega, u(\omega) ; d) \leq f(\omega, u(\omega)+d)-f(\omega, u(\omega)) .
\end{aligned}
$$

Since $f(\omega, \cdot)$ is continuous, for every order convex neighborhood $V$ of the origin in $Y$, there is a $\delta>0$ s.t. if $\|d\|<\delta$ we have

$$
f(\omega, u(\omega))-f(\omega, u(\omega)-d), \quad f(\omega, u(\omega)+d)-f(\omega, u(\omega)) \in V .
$$

Then using $(*)$ and the order convexity in $V$, we can conclude that, for all $\|d\|<\delta, f^{\prime}(\omega, u(\omega) ; d) \in V$, which shows that $f(\omega, \mathrm{u}(\omega) ; \cdot)$ is continuous at the origin. Next, using the sublinearity of $f^{\prime}(\omega, u(\omega) ; \cdot)$, we will show that the continuity is in fact global. So let $d \in X \backslash\{0\}$ and let $d_{n} \stackrel{\text { s }}{\rightarrow} d$. Then because of the sublinearity of $f(\omega, u(\omega) ; \cdot)$, we have

$$
\begin{aligned}
& \left|f\left(\omega, u(\omega) ; d_{n}\right)-f(\omega, u(\omega) ; d)\right| \\
& \quad \leq f\left(\omega, u(\omega) ; d_{n}-d\right) \vee f\left(\omega, u(\omega) ; d-d_{n}\right),
\end{aligned}
$$

so

$$
\begin{aligned}
& \left\|f\left(\omega, u(\omega) ; d_{n}\right)-f(\omega, u(\omega) ; d)\right\| \\
& \quad \leq\left\|f\left(\omega, u(\omega) ; d_{n}-d\right) \vee f\left(\omega, u(\omega) ; d-d_{n}\right)\right\| .
\end{aligned}
$$

Since the lattice operations are strongly continuous,

$$
\lim _{n \rightarrow \infty}\left\|f\left(\omega, u(\omega) ; d_{n}\right)-f(\omega, u(\omega) ; d)\right\|=0 .
$$

Hence $f(\omega, u(\omega) ; \cdot)$ is continuous on all $X$ as claimed. Now by $S(\cdot)$ we denote the multifunction $\omega \rightarrow \partial f(\omega, u(\omega))$. Then

$$
S(\omega)=\left\{A \in \mathscr{L}(X, Y): A(d) \leq f^{\prime}(\omega, u(\omega) ; d) \text { for all } d \in X\right\} .
$$

Note that if for some $A \in \mathscr{L}(X, Y)$, we have $A\left(d_{n}\right) \leq f^{\prime}\left(\omega, u(\omega) ; d_{n}\right)$, where $\left\{d_{n}\right\}_{n \geq 1}$ is a dense subset of $X$, then from the continuity of $f^{\prime}(\omega, u(\omega) ; \cdot)$ and $A(\cdot)$, and also from the fact that $Y_{+}$is closed, we can conclude that $A(d) \leq f(\omega, u(\omega)$; $d)$ for all $d \in X$, so $A \in \partial f(\omega, u(\omega))$. So for $n \geq 1$, consider the operators $g_{n}: \Omega \times \mathscr{L}(X, Y) \rightarrow Y$ defined by $g_{n}(\omega, A)=A\left(d_{n}\right)-f^{\prime}\left(\omega, u(\omega) ; d_{n}\right)$. These are jointly measurable. Let $G_{n}=\left\{(\omega, A) \in \Omega \times \mathscr{L}(X, Y): g_{n}(\omega, A) \leq 0\right\}$. Then for all $n \geq 1, G_{n} \in$ $\Sigma \times B(X)$. But observe that Gr $S=\bigcap_{n \geq 1} G_{n}$. So Gr $S \in \Sigma \times B(X)$.

REMARK. If $X, Y$ are finite dimensional, then $\mathscr{L}(X, Y)$ is separable, so from Theorem 3.4 and the fact that the subdifferential is closed valued, we 
can conclude, using Theorem 2.1, that $\omega \rightarrow \partial f(\omega, u(\omega))$ is measurable for all $u(\cdot) \in L_{X}^{P}(\Omega)$.

However, we can show that another multifunction originating from the integrand operator $f(\cdot, \cdot)$, namely the epigraph multifunction, is measurable.

Recall that for every $\omega \in \Omega$, the epigraph of $f(\omega, \cdot)$ is defined by

$$
\text { epi } f(\omega, \cdot)=\{(x, y) \in X \times Y: f(\omega, x) \leq y\} \text {. }
$$

Since $f(\cdot, \cdot)$ is Caratheodory, it is easy to see that $\omega \rightarrow$ epi $f(\omega, \cdot)$ is closed valued. (It is also convex if $f(\omega, \cdot)$ is convex, but we do not need this for the next theorem.)

THEOREM 3.5. The multifunction $\omega \rightarrow$ epi $f(\omega, \cdot)$ is measurable.

Proof. By definition

$$
\text { epi } \begin{aligned}
f(\omega, \cdot) & =\{(x, y) \in X \times Y: f(\omega, x) \leq y\} \\
& =\{(x, y) \in X \times Y: f(\omega, x)-y \leq 0\} .
\end{aligned}
$$

Let $f(\omega, x)-y=\phi(\omega, x, y)$. Clearly $\phi$ is measurable in $\omega$ and continuous in $(x, y)$. Hence using Theorem 6.4 of [9] (or Lemma III-14 of [4]), we conclude that $\omega \rightarrow$ epi $f(\omega, \cdot)$ is measurable as claimed.

The next result compares the o-directional derivatives of two Caratheodory convex integrand operators $f(\cdot, \cdot)$ and $g(\cdot, \cdot)$ which satisfy a certain integral equality.

THEOREM 3.6. If, for every $u(\cdot) \in L_{X}^{\mathcal{f}}(\Omega)$, we have

$$
\int_{\Omega} f(\omega, u(\omega)) d \mu(\omega)=\int_{\Omega} g(\omega, u(\omega)) d \mu(\omega),
$$

then for every $h(\cdot) \in L_{X}^{p}(\Omega)$, we have

$$
\int_{\Omega} f^{\prime}(\omega, u(\omega) ; h(\omega)) d \mu(\omega)=\int_{\Omega} g^{\prime}(\omega, u(\omega) ; h(\omega)) d \mu(\omega) .
$$

Proof. By definition

$$
\begin{aligned}
\int_{\Omega} f^{\prime}(\omega, u(\omega) ; h(\omega)) d \mu(\omega) \\
=\int_{\Omega} \underset{\lambda_{n} \downarrow 0}{\operatorname{o-lim}} \frac{f\left(\omega, u(\omega)+\lambda_{n} h(\omega)\right)-f(\omega, u(\omega))}{\lambda_{n}} d \mu(\omega) .
\end{aligned}
$$


From Lemma 8 of Valadier [24], we know that

$$
\begin{aligned}
\mathrm{o}-\lim _{n \rightarrow \infty} & \frac{f\left(\omega, u(\omega)+\lambda_{n} h(\omega)\right)-f(\omega, u(\omega))}{\lambda_{n}} \\
= & \operatorname{w}_{n \rightarrow \infty} \frac{f\left(\omega, u(\omega)+\lambda_{n} h(\omega)\right)-f(\omega, u(\omega))}{\lambda_{n}} .
\end{aligned}
$$

So for every $y^{*} \in Y^{*}$,

$$
\begin{aligned}
& \int_{\Omega} f^{\prime}(\omega,u(\omega) ; h(\omega)) d \mu(\omega) \\
&=\int_{\Omega} \lim _{n \rightarrow \infty}\left(y^{*}, \frac{f\left(\omega, u(\omega)+\lambda_{n} h(\omega)\right)-f(\omega, u(\omega))}{\lambda_{n}}\right) d \mu(\omega),
\end{aligned}
$$

and from the Lebesgue dominated convergence theorem we know we can pull the limit outside the integral. So,

$$
\begin{aligned}
\int_{\Omega} f^{\prime}(\omega, & u(\omega) ; h(\omega)) d \mu(\omega) \\
= & \lim _{n \rightarrow \infty} \int_{\Omega}\left(y^{*}, \frac{f\left(\omega, u(\omega)+\lambda_{n} h(\omega)\right)-f(\omega, u(\omega))}{\lambda_{n}}\right) d \mu(\omega) \\
= & \lim _{n \rightarrow \infty}\left(y^{*}, \int_{\Omega} \frac{f\left(\omega, u(\omega)+\lambda_{n} h(\omega)\right)-f(\omega, u(\omega))}{\lambda_{n}}\right) d_{\mu}(\omega) \\
= & \lim _{n \rightarrow \infty}\left(y^{*}, \int_{\Omega} \frac{g\left(\omega, u(\omega)+\lambda_{n} h(\omega)\right)-g(\omega, u(\omega))}{\lambda_{n}}\right) d \mu(\omega) .
\end{aligned}
$$

Following the same reasoning as above, we easily obtain

$$
\begin{gathered}
\lim _{n \rightarrow \infty}\left(y^{*}, \int_{\Omega} \frac{g\left(\omega, u(\omega)+\lambda_{n} h(\omega)\right)-g(\omega, u(\omega))}{\lambda_{n}}\right) d \mu(\omega) \\
=\int_{\Omega} g^{\prime}(\omega, u(\omega) ; h(\omega)) d \mu(\omega) .
\end{gathered}
$$

Therefore we conclude that

$$
\int_{\Omega} f^{\prime}(\omega, u(\omega) ; h(\omega)) d \mu(\omega)=\int_{\Omega} g^{\prime}(\omega, u(\omega) ; h(\omega)) d \mu(\omega) .
$$

A useful notion in convex analysis, that provides us a better understanding of the asymptotic behavior of a convex function, is that of the recession function. If we start with a convex operator $f: X \rightarrow Y$, then its asymptotic operator $f 0^{+}$is the operator that has as its epigraph the asymptotic cone of epi $f$. This definition immediately leads us to the conclusion that $\left(f 0^{+}\right)(\cdot)$ is a positively homogeneous convex operator. 
Furthermore, if $f(\cdot)$ is continuous, then $f 0^{+}$is given by

$$
\left(f 0^{+}\right)(h)=\sup _{\lambda>0} \frac{f(x+\lambda h)-f(x)}{\lambda} .
$$

Since the quotients are increasing with $\lambda$, we can write

$$
\left(f 0^{+}\right)(h)=\underset{\lambda \rightarrow \infty}{o-\lim } \frac{f(x+\lambda h)-f(x)}{\lambda} .
$$

If $f(\cdot)$ is order bounded form above, then, employing once more Lemma 8 of Valadier [24], we can show that

$$
\left(f 0^{+}\right)(h)=\underset{\lambda \rightarrow \infty}{\mathrm{w}-\lim } \frac{f(x+\lambda y)-f(x)}{\lambda} .
$$

In fact, and the same is true for the o-directional derivative, we can show, using some results of [23], that the limit can actually be taken in the norm topology. However, the weak convergence is enough for our purposes.

Then using $(*)$ and a proof parallel to that of Theorem 3.6, we can easily prove the following result. As always, $f: \Omega \times X \rightarrow Y$ is a Caratheodory convex integrand operator.

THEOREM 3.7. If there is a $g(\cdot) \in L_{Y}^{1}(\Omega)$ s.t. $|f(\omega, x)| \leq g(\omega) \mu$-a.e. for all $x \in X$, then for all $h(\cdot) \in L_{X}^{p}(\Omega)$,

$$
\left(F 0^{+}\right)(h)=\int_{\Omega}\left(f 0^{+}\right)(\omega ; h(\omega)) d \mu(\omega) .
$$

4. Conditional expectations. In this section we study the conditional expectation of Caratheodory convex integrand operators. In sequential multiobjective decision making, such as multistage stochastic programming problems, we work with the observations (information) that we gather as the process evolves and so we need to consider the conditional expectation of the cost integrand $f(\cdot, \cdot)$.

So suppose $f(\cdot, \cdot): \Omega \times X \rightarrow Y$ is a Caratheodory (convex) integrand operator s.t. $f(\cdot, x) \in L_{Y}^{1}(\Omega)$ for all $x \in X$. Let $\Sigma_{0}$ be a sub $\sigma$-field of $\Sigma$.

Definition 4.1. We say that $g: \Omega \times X \rightarrow Y$ is a $\Sigma_{0}$-conditional expectation of $f(\cdot, \cdot)$ if and only if $g(\cdot, \cdot)$ is a Caratheodory (convex) integrand operator, for all $x \in X, g(\cdot, x) \in L_{Y}^{1}\left(\Omega, \Sigma_{0}\right)$ and for all $A \in \Sigma_{0}$,

$$
\int_{A} f(\omega, x) d \mu(\omega)=\int_{A} g(\omega, x) d \mu(\omega),
$$

i.e. for all $x \in X, g(\cdot, x)=E^{\Sigma_{0}} f(\cdot, x)$. 
Naturally enough we start with an existence and uniqueness result.

THEOREM 4.1. If $f: \Omega \times X$ is as above then $f(\cdot, \cdot)$ has a $\Sigma_{0}$-conditional expectation which is unique up to $\mu$-a.e. equality.

Proof. Since for every $x \in X, f(\cdot, x) \in L_{Y}^{1}(\Omega)$, we know from Theorem 4 of [6] that $g(\cdot, x)=E^{\Sigma_{0}} f(\cdot, x)$ exists and is unique up to $\mu$-a.e. equality, for $x \in D=$ dense in $X$ we claim that for $\mu$-almost all $\omega \in \Omega$ $x \rightarrow g(\omega, x)$ is continuous. So fix an $\omega \in \Omega$ and let $\varepsilon>0$. Then there is a $\delta>0$ s.t. if $\left\|x-x^{\prime}\right\|<\delta$, where $x^{\prime}, x \in D$, we have $\left\|f(\omega, x)-f\left(\omega, x^{\prime}\right)\right\|$ $<\varepsilon$. Also we have

$$
\begin{aligned}
\left\|g(\omega, x)-g\left(\omega, x^{\prime}\right)\right\| & =\left\|E^{\Sigma_{0}} f\left(\omega, x^{\prime}\right)-E^{\Sigma_{0}} f(\omega, x)\right\| \\
& =\left\|E^{\Sigma_{0}}\left(f(\omega, x)-f\left(\omega, x^{\prime}\right)\right)\right\| .
\end{aligned}
$$

From Jensen's inequality, we have

$$
\left\|E^{\Sigma_{0}}\left(f(\omega, x)-f\left(\omega, x^{\prime}\right)\right)\right\| \leq E^{\Sigma_{0}}\left\|f(\omega, x)-f\left(\omega, x^{\prime}\right)\right\| .
$$

So eventually we get

$$
\left\|g(\omega, x)-g\left(\omega, x^{\prime}\right)\right\| \leq E^{\Sigma_{0}}\left\|f(\omega, x)-f\left(\omega, x^{\prime}\right)\right\| \leq E^{\Sigma_{0}} \varepsilon=\varepsilon,
$$

which proves that $g(\omega, \cdot)$ is continuous on $D$. It is also $o$-Lipschitz [13] and so extends uniquely to $X$ and $E^{\Sigma_{0}} f(\omega, x)=g(\omega, x)$.

Next for all $x_{1}, x_{2} \in X$ and $\lambda \in(0,1)$ and for all $A \in \Sigma_{0}$, we have, from the convexity of $f(\omega, \cdot)$ and Lemma $\alpha$,

$$
\begin{aligned}
\int_{A} g(\omega, & \left.\lambda x_{1}+(1-\lambda) x_{2}\right) d \mu(\omega)=\int_{A} f\left(\omega, \lambda x_{1}+(1-\lambda) x_{2}\right) d \mu(\omega) \\
\leq & \int_{A}\left(\lambda f\left(\omega, x_{1}\right)+(1-\lambda) f\left(\omega, x_{2}\right)\right) d \mu(\omega) \\
& =\int_{A}\left(\lambda g\left(\omega, x_{1}\right)+1(1-\lambda) g\left(\omega, x_{2}\right)\right) d \mu(\omega)
\end{aligned}
$$

So, for every $y^{*} \in Y_{+}^{*}$,

$$
\begin{aligned}
\left(y^{*}, \int_{A}\right. & \left.g\left(\omega, \lambda x_{1}+(1-\lambda) x_{2}\right) d \mu(\omega)\right) \\
\quad & \int_{A}\left(y^{*}, g\left(\omega, \lambda x_{1}+(1-\lambda) x_{2}\right)\right) d \mu(\omega) \\
\leq & \left(y^{*} \int_{A}\left(\lambda g\left(\omega, x_{1}\right)+(1-\lambda) g\left(\omega, x_{2}\right)\right) d \mu(\omega)\right) \\
\quad= & \int_{A}\left(y^{*}, \lambda g\left(\omega, x_{1}\right)+(1-\lambda) g\left(\omega, x_{2}\right)\right) d \mu(\omega) .
\end{aligned}
$$


Hence

$$
\begin{aligned}
& \left(y^{*}, g\left(\omega, \lambda x_{1}+(1-\lambda) x_{2}\right)\right) \\
& \quad \leq\left(y^{*}, \lambda g\left(\omega, x_{1}\right)+(1-\lambda) g\left(\omega, x_{2}\right)\right) \quad \mu \text {-a.e. }
\end{aligned}
$$

But from Krein's theorem (see [16], p. 72), we know that $Y_{+}^{*}$ is generating, i.e. $Y^{*}=Y_{+}^{*}-Y_{+}^{*}$. Using that, we get

$$
g\left(\omega, \lambda x_{1}+(1-\lambda) x_{2}\right) \leq \lambda g\left(\omega, x_{1}\right)+(1-\lambda) g\left(\omega, x_{2}\right) \quad \mu \text {-a.e. }
$$

By changing $g(\cdot, \cdot)$ on a set of $\mu$-measure zero we get that $g(\omega, \cdot)$ is convex for all $\omega \in \Omega$.

Actually from both a theoretical and applied viewpoint we would like to know something stronger than the previous result. Namely, whether the $\Sigma_{0}$-conditional expectation is uneffected when we compose $f(\omega, \cdot)$ with an element in $L_{X}^{p}\left(\Omega ; \Sigma_{0}\right)$. So now let $f(\cdot, \cdot)$ be an $L^{1}(\Omega)$-bounded Caratheodory convex integrand.

THEOREM 4.2. If $g \in E^{\Sigma_{0}} f$ then for all $u(\cdot) \in L_{X}^{p}\left(\Omega, \Sigma_{0}\right), g(\omega, u(\omega))$ is the $\Sigma_{0}$ conditional expectation of $f(\omega, u(\omega))$.

Proof. Since by hypothesis $g=E^{\Sigma_{0}} f$ for every $x \in X$, and for every $A \in \Sigma_{0}$ we have

$$
\int_{A} f(\omega, x) d \mu(\omega)=\int_{A} g(\omega, x) d \mu(\omega)
$$

Now consider a simple function $s(\omega)=\sum_{k=1}^{n} x_{k} \chi_{A_{k}}(\omega)$ with $A_{k} \in \Sigma_{0}$, $k=1, \ldots, n$. For such a function we have

$$
\begin{aligned}
\int_{A} f( & \omega, s(\omega)) d \mu(\omega)=\int_{A} f\left(\omega, \sum_{k=1}^{n} x_{k} \chi_{A_{k}}(\omega)\right) d \mu(\omega) \\
= & \sum_{k=1}^{n} \int_{A \cap A_{k}} f\left(\omega, x_{k}\right) d \mu(\omega)=\sum_{k=1}^{n} \int_{A \cap A_{k}} g\left(\omega, x_{k}\right) d \mu(\omega) \\
= & \int_{A} g\left(\omega, \sum_{k=1}^{n} x_{k} \chi_{A_{k}}(\omega)\right) d \mu(\omega)=\int_{A} g(\omega, s(\omega)) d \mu(\omega) .
\end{aligned}
$$

But we know from [6] that we can find simple functions $\left\{s_{n}\right\}_{n \geq 1}$ s.t. $s_{n}(\omega) \stackrel{s}{\rightarrow} u(\omega) \mu$-a.e. Then from the Lebesgue dominated convergence 
theorem and the continuity of $f(\omega, \cdot)$ and $g(\omega, \cdot)$, we get

$$
\begin{aligned}
\lim _{n \rightarrow \infty} \int_{A} f\left(\omega, s_{n}(\omega)\right) d \mu(\omega) \\
\quad=\int_{A} \lim _{n \rightarrow \infty} f\left(\omega, s_{n}(\omega)\right) d \mu(\omega)=\int_{A} f(\omega, u(\omega)) d \mu(\omega)
\end{aligned}
$$

and

$$
\begin{aligned}
\lim _{n \rightarrow \infty} \int_{A} g\left(\omega, s_{n}(\omega)\right) d \mu(\omega) \\
\quad=\int_{A} \lim _{n \rightarrow \infty} g\left(\omega, s_{n}(\omega)\right) d \mu(\omega)=\int_{A} g(\omega, u(\omega)) d \mu(\omega) .
\end{aligned}
$$

(Note that for every $x \in X$,

$$
\left.\|g(\omega, x)\|=\left\|E^{\Sigma_{0}} f(\omega, x)\right\| \leq E^{\Sigma_{0}}\|f(\omega, x)\| \leq E^{\Sigma_{0}} \phi(\omega) \quad \text { a.e.. }\right)
$$

Therefore for all $A \in \Sigma_{0}$,

$$
\int_{A} f(\omega, u(\omega)) d \mu(\omega)=\int_{A} g(\omega, u(\omega)) d \mu(\omega) .
$$

Using this result, we can easily show that for all $u(\cdot), h(\cdot) \in L_{X}^{p}(\Omega)$,

$$
E^{\Sigma_{0}} f^{\prime}(\omega, u(\omega) ; h(\omega))=g^{\prime}(\omega, u(\omega) ; h(\omega)) .
$$

This can be viewed as a completion to Theorem 3.6.

We can generalize our result on the existence of $\Sigma_{0}$-conditional expectations to (convex) integrand operators that are in $L_{Y}^{1}(\Omega)$ only locally. However, before doing that we need to introduce some auxiliary material. To our range space $Y$, we adjoin a greatest element $+\infty$ and extend the vector space operations in a natural way. Therefore, we set $+\infty+(-\infty)=+\infty$. We will denote the augmented space $Y \cup\{ \pm \infty\}$ by $\hat{Y}$. The topology of $Y$ is then extended to $\hat{Y}$ as follows. A set $\hat{U}$ will be said to be open in $\hat{Y}$ if the trace of $\hat{U}$ on $Y$ is open and if $-\infty \in \hat{U}$ (resp. $+\infty \in \hat{U}$ ); then for $U=\hat{U} \cap Y$ we have $U-Y_{+}=U$ (resp. $U=U+$ $\left.Y_{+}\right)$. We have then to extend the norm of $Y$ to include $\pm \infty$. So we define $\| \pm \infty\|=\infty$ and we say that $y_{n} \stackrel{s}{\rightarrow}+\infty$ if and only if $\left\|y_{n}^{+}\right\| \rightarrow \infty$ and $\left\{\left\|y_{n}^{-}\right\|\right\}_{n \geq 1}$ is bounded, while $y_{n} \stackrel{s}{\rightarrow}-\infty$ if and only if $\left\|y_{n}^{-}\right\| \rightarrow \infty$ and $\left\{\left\|y_{n}^{+}\right\|\right\}_{n \geq 1}$ is bounded. This definition is consistent with the extended topology defined earlier, since, as we can easily check, the sets $\hat{U}_{M}=$ $\left\{y \in Y:\left\|y^{+}\right\|>M_{1},\left\|y^{-}\right\| \leq M_{2}\right\}$ and $\hat{V}_{M}=\left\{y \in Y:\left\|y^{-}\right\|>M_{1},\left\|y^{+}\right\| \leq\right.$ $\left.M_{2}\right\}, M_{1}, M_{2}>0$, are neighborhoods of $+\infty$ and $-\infty$, respectively, in 
that topology. The introduction of these points at infinity is very useful for purposes of nonsmooth analysis and optimization. Among other things, it allows us to incorporate the constraints of a constraint vectorial optimization problem into the cost operator using the indicator function and so to transform the problem into one without constraints.

In the next result we will consider operators with values in $\hat{Y}$. So we have

THEOREM 4.3 If $f: \Omega \times X \rightarrow \hat{Y}$ is a (convex) measurable operator s.t.:

(i) $\sup _{x \in n B(0,1)}\|f(\omega, x)\| \leq \alpha_{n}(\omega)$, where $\alpha_{n}(\cdot) \in L^{1}(\Omega), n \geq 1$, and $B(0,1)=\{x \in X:\|x\| \leq 1\}$

(ii) for all $x \in X, f^{-}(\cdot, x) \in L_{Y}^{1}(\Omega)$ then $f(\cdot, \cdot)$ has a unique $\Sigma_{0}$-conditional expectation in the sense that there is a unique (convex) integrand operator $g: \Omega \times X \rightarrow Y$ s.t. for all $A \in \Sigma_{0}$ and all $x(\cdot) \in L_{X}^{\infty}(0)$,

$$
\int_{A} f(\omega, x(\omega)) d \mu(\omega)=\int_{A} g(\omega, x(\omega)) d \mu(\omega),
$$

where the above integrals may be $+\infty$.

Proof. For $n \geq 1$ and for a fixed $y \in Y_{+}$, consider the integrand operators

$$
f_{n}(\omega, x)= \begin{cases}f(\omega, x), & x \in n B_{1}, \\ y \wedge f(\omega, x) & x \notin n B_{1}\end{cases}
$$

Clearly, $f_{n}(\omega, x) \uparrow f(\omega, x)$ as $n \rightarrow \infty$. Since $Y$ is separable, so is $\hat{Y}$, and then from Schaefer [23] we know that the latter has an order continuous norm. So $f_{n}(\omega, n) \stackrel{s}{\rightarrow} f(\omega, x)$. Observe that for all $n \geq 1$ and $x \in X$, $f_{n}(\cdot, x) \in L_{Y}^{1}(\Omega)$. So $f_{n}(\cdot, x)$ has a $\Sigma_{0}$-conditional expectation which we denote by $g_{n}(\cdot, x) \in L_{Y}^{1}\left(\Omega ; \Sigma_{0}\right)$. Hence, for all $A \in \Sigma_{0}$ and all $x(\cdot) \in$ $L_{X}^{\infty}(0)$

$$
\begin{aligned}
& \int_{A} f_{n}(\omega, x(\omega)) d \mu(\omega)=\int_{A} g_{n}(\omega, x(\omega)) d \mu(\omega) \\
& \quad \leq \int_{A} f_{n+1}(\omega, x(\omega)) d \mu(\omega)=\int_{A} g_{n+1}(\omega, x(\omega)) d \mu(\omega),
\end{aligned}
$$

which implies $g_{n}(\omega, x) \leq g_{n+1}(\omega, x) \mu$-a.e. So we see that $\left\{g_{n}(\cdot, x)\right\}_{n \geq 1}$ is increasing sequence of $\hat{Y}$-valued operators for all $x \in X$. Let $g(\omega, x)=$ $\bigvee_{n=1}^{\infty} g_{n}(\omega, x)$. Because the measurable operators from $\Omega$ into $Y$ are an order complete lattice, we deduce that $g(\cdot, x)$ is measurable for every 
$x \in X$. Also observe that, for all $y^{*} \in Y_{+}^{*}$ and all $A \in \Sigma_{0}$,

$$
\begin{aligned}
& \left(y^{*}, \int_{A} f_{n}(\omega, x) d \mu(\omega)\right) \\
& \quad=\int_{A}\left(y^{*}, f_{n}(\omega, x)\right) d \mu(\omega) \uparrow \int_{A}\left(y^{*}, f(\omega, x)\right) d \mu(\omega) \\
& \quad=\left(y^{*}, \int_{A} f(\omega, x) d \mu(\omega)\right) .
\end{aligned}
$$

Similarly, we get

$$
\left(y^{*}, \int_{A} g_{n}(\omega, x) d \mu(\omega)\right) \uparrow\left(y^{*}, \int_{A} g(\omega, x) d \mu(\omega)\right) .
$$

We consider two cases: have

I If $\int_{A} f(\omega, x) d \mu(\omega)=+\infty$ then $\left(y^{*}, \int_{A} g(\omega, x)\right)=+\infty$. So we

$$
\int_{A} f(\omega, x) d \mu(\omega)=\int_{A} g(\omega, x) d \mu(\omega)=+\infty .
$$

II If $\int_{A} f(\omega, x) d \mu(\omega)<+\infty$ then, since by Krein's theorem $Y_{1}^{*}$ is generating,

$$
\int_{A} f(\omega, x) d \mu(\omega)=\int_{A} g(\omega, x) d \mu(\omega) .
$$

If $f(\cdot, \cdot)$ is, in addition, convex, then, as in Theorem 4.1, we can show that $g(\cdot, \cdot)$ is also. Hence $E^{\Sigma_{0}} f=g$.

Now that we have established the existence of a $\Sigma_{0}$-conditional expectation of a Caratheodory convex integrand operator in the sense of Definition 4.1, we will proceed and compare their subdifferentials.

THEOREM 4.4. If $\mathscr{L}(X, Y)$ has the Radon-Nikodym property, $f: \Omega \times X$ $\rightarrow Y$ is an $L^{1}(\Omega)$-bounded Caratheodory convex integrand operator, and $g=E^{\Sigma_{0}} f$, then $R(\cdot) \in \partial g(\omega, x)$ if and only if there exists $T(\cdot) \in \partial f(\omega, x)$ s.t. for all $h(\cdot) \in L_{X}^{p}(\Omega)$,

$$
E^{\Sigma_{0}} T(\omega, h(\omega))=R(\omega, h(\omega)) \quad \mu \text {-a.e. }
$$

Proof. First let $R(\cdot) \in \partial g(\cdot, x)$. Then $R(\omega) \in \partial g(\omega, x) \mu$-a.e. so $R(\omega, h(\omega)) \leq g^{\prime}(\omega, x ; h(\omega)) \mu$-a.e. for all $h(\cdot) \in L_{X}^{p}(\Omega)$. But as we mentioned earlier in this section,

$$
\int_{\Omega} g^{\prime}(\omega, x ; h(\omega)) d \mu(\omega)=\int_{\Omega} f^{\prime}(\omega, x ; h(\omega)) d \mu(\omega)
$$


Let $R(h)=\int_{\Omega} R(\omega, h(\omega)) d \mu(\omega)$. This is linear and continuous for the norm topology on $L_{X}^{p}(\Omega)$. So from the result of Neumann [11] and Saint Pierre [22], we know there exists $T(\cdot) \in L_{\mathscr{L}(X, Y)}^{1}(\Omega, \Sigma)$ s.t.

$$
R(h)=\int_{\Omega} T(\omega, h(\omega)) d \mu(\omega)
$$

and

$$
T(\omega, h(\omega)) \leq f^{\prime}(\omega, x ; h(\omega)) \quad \mu \text {-a.e. }
$$

Observe that the latter inequality implies that $T(\omega) \in \partial f(\omega, x) \mu$-a.e. so $T(\cdot) \in \partial f(\cdot, x)$.

Now let $h(\omega)=\hat{h}(\omega) \chi_{A}(\omega)$, where $A \in \Sigma_{0}$ and $\hat{h}(\cdot) \in L_{X}^{p}\left(\Omega, \Sigma_{0}\right)$. Then

$$
R(h)=\int_{\Omega} T\left(\omega, \hat{h}(\omega) \chi_{A}(\omega)\right) d \mu(\omega)=\int_{\Omega} R\left(\omega, \hat{h}(\omega) \chi_{A}(\omega)\right) d \mu(\omega),
$$

which implies

$$
\int_{A} T(\omega, \hat{h}(\omega)) d \mu(\omega)=\int_{A} R(\omega, \hat{h}(\omega)) d \mu(\omega),
$$

and since this is true for all $A \in \Sigma_{0}$, we conclude that

$$
E^{\Sigma_{0}} T(\omega, \hat{h}(\omega))=R(\omega, \hat{h}(\omega)) \quad \mu \text {-a.e. }
$$

as claimed.

The implication in the opposite direction is more or less obvious, because if we let $T(\cdot) \in \partial f(\cdot, x)$ and $R(\omega, h)=E^{\Sigma_{0}} T(\omega, h) \mu$-a.e., since $T(\cdot) \in \partial f(\cdot, x)$, we know that for all $h \in X$,

$$
T(\omega, h) \leq f^{\prime}(\omega, x ; h),
$$

and integrating over any $A \in \Sigma_{0}$, we get

$$
\int_{A} T(\omega, h) d \mu(\omega) \leq \int_{A} f^{\prime}(\omega, x ; h) d \mu(\omega) .
$$

But once again, we use the fact that

$$
\int_{A} f^{\prime}(\omega, x ; h) d \mu(\omega)=\int_{A} g^{\prime}(\omega, x ; h) d \mu(\omega) .
$$

Also since $E^{\Sigma_{0}} T(\omega, h)=R(\omega, h) \mu$-a.e., we have

$$
\int_{A} T(\omega, h) d \mu(\omega)=\int_{A} R(\omega, h) d \mu(\omega) .
$$


Combining (1), (2) and (3) above, we get

$$
\int_{A} R(\omega, h) d \mu(\omega) \leq \int_{A} g^{\prime}(\omega, x ; h) d \mu(\omega)
$$

for all $A \in \Sigma_{0}$ and all $h \in X$. Since both integrands are $\Sigma_{0}$-measurable we get that for all $h \in X, R(\omega, h) \leq g^{\prime}(\omega, x ; h) \mu$-a.e., which means $R(\omega) \in$ $\partial g(\omega, x) \mu$-a.e. So $R(\cdot) \in \partial g(\cdot, x)$.

In the next result, we examine the conditional expectation of the other important multifunction associated with $f$, namely the epigraph of $f$. For that purpose, assume $X$ and $Y$ are, in addition, reflexive and $f: \Omega \times X \rightarrow Y$ is a Caratheodory convex integrand operator s.t. for all $x \in X,\|f(\omega, x)\|$ $\leq \phi(\omega) \mu$-a.e., $\phi(\cdot) \in L^{\infty}(\Omega)$. Assume $X, Y$ are locally uniformly convex.

THEOREM 4.5. If $g \in E^{\Sigma_{0}} f$ then $E^{\Sigma_{0}}$ epi $f(\omega, \cdot)$ exists and

$$
E^{\Sigma_{0}} \text { epi } f(\omega, \cdot) \subseteq \text { epi } g(\omega, \cdot) \text {. }
$$

REMARK. Note that by its nature epi $f(\omega, \cdot)$ is not integrably bounded so the existence result cannot be deduced from the work of Hiai-Umegaki [8].

Proof. To prove the existence of $E^{\Sigma_{0}}$ epi $f(\omega, \cdot)$, we will use an approximation method. So for $n \geq 1$, let epi $f^{n}(\omega, \cdot)=\operatorname{epi} f(\omega, \cdot) \cap$ $n B(0,1)$. Clearly, epi $f^{n}(\omega, \cdot)$ is integrably bounded, so by Hiai-Umegaki [8], we know that $E^{\Sigma_{0}}$ epi $f^{n}(\omega, \cdot)$ exists and is a closed, convex valued, integrably bounded multifunction.

Note that epi $f^{n}(\omega, \cdot) \uparrow$ epi $f(\omega, \cdot)$ as $n \rightarrow \infty$ for all $\omega \in \Omega$. Then for all $z^{*} \in Z^{*}=(X \times Y)^{*}=X^{*} \times Y^{*}, \sigma_{\text {epi } f^{n}(\omega,)}\left(z^{*}\right) \uparrow \sigma_{\text {epi } f(\omega,)}\left(z^{*}\right)$. Also for $n>m \geq 1$, since epi $f^{n}(\omega, \cdot)$ and epi $f^{m}(\omega, \cdot)$ are both bounded, closed and convex, we can use Hörmander's formula and obtain

$$
\begin{aligned}
h\left(\text { epi } f^{n}\right. & \left.(\omega, \cdot), \text { epi } f^{m}(\omega, \cdot)\right) \\
& =\sup _{\left\|z^{*}\right\|=1}\left|\sigma_{\text {ep } f^{n}(\omega, \cdot)}\left(z^{*}\right)-\sigma_{\text {epi } f^{m}(\omega, \cdot)}\left(z^{*}\right)\right| \\
& \leq \sup _{\left\|z^{*}\right\|=1}\left|\sigma_{\text {epi } f^{m}(\omega,) \cap\left[\operatorname{epi} f^{m}(\omega, \cdot)\right]^{c}}\left(z^{*}\right)\right| \\
& =\sup _{\left\|z^{*}\right\|=1}\left|\sigma_{\left.\overline{\text { conv }} \operatorname{epi} f^{m}(\omega,)\right) \cap\left[\operatorname{epi} f^{m}(\omega, \cdot)\right]^{c}}\left(z^{*}\right)\right| .
\end{aligned}
$$

Then by Aubin's [1] Proposition 6 (p. 99), we know that

$$
\sigma_{\overline{\text { conv }} \text { ep } 1} f^{m}(\omega,) \cap\left[\text { epi } f^{m}(\omega, \cdot)\right]^{c}(\cdot)
$$


is norm continuous on $Z^{*}$. Since $Z=X \times Y$ is reflexive, by Troyansky's renorming theorem (see [6]), we can assume without loss of generality that $Z$ is locally uniformly convex. Then suppose $z_{n} \stackrel{\text { w }}{\rightarrow} z$ with $\left\|z_{n}\right\|=\|z\|=1$ for all $n \geq 1$. By local uniform convexity, $z_{n} \stackrel{\mathrm{s}}{\rightarrow} z$ so

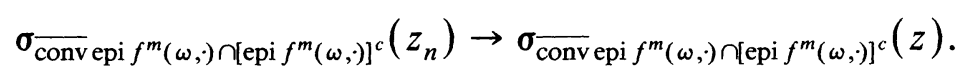

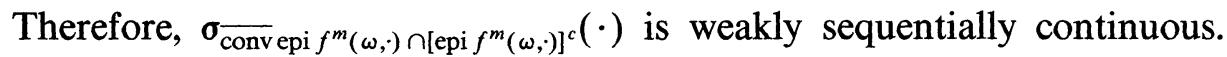
Hence from the weak sequential compactness of the unit ball, we can see that there exists $z^{*} \in Z^{*}$ (depending on $\omega \in \Omega$ ) s.t. $\left\|\hat{z}^{*}\right\|=1$, for which the supremum in $(*)$ is attained. So we have, for all $\omega \in \Omega$,

$$
h\left(\text { epi } f^{n}(\omega, \cdot) \text {, epi } f^{m}(\omega, \cdot)\right)=\sigma \overline{\operatorname{conv}}\left(\text { epi } f^{n}(\omega,)\right) \Upsilon\left[\text { epi } f^{m}(\omega,)\right]^{c}\left(\hat{z}^{*}\right) \rightarrow 0
$$

as $n, m \rightarrow \infty$.

Therefore for every $\omega \in \Omega$ epi $\left.f^{n}(\omega, \cdot)\right\}_{n \geq 1}$ is an $h$-Cauchy sequence in $P_{f c}(Z)$. But recall that the latter endowed with the Hausdorff metric is complete. So for all $\omega \in \Omega$, epi $f^{n}(\omega, \cdot) \rightarrow S(\omega) \in P_{f c}(X)$. Also, using the homogeneity of the support functional, $\sigma_{\text {epi } f^{n}(\omega,)}\left(z^{*}\right) \rightarrow \sigma_{S(\omega)}\left(z^{*}\right)$ for all $z^{*} \in Z^{*}$ and all $\omega \in \Omega$. But we already saw in the beginning of the proof that $\sigma_{\text {epi } f^{n}(\omega,)}\left(z^{*}\right) \rightarrow \sigma_{\text {epi } f(\omega,)}\left(z^{*}\right)$ for all $z^{*} \in Z^{*}$ and all $\omega \in \Omega$. Hence,

$$
\sigma_{\text {epi } f(\omega,)}\left(z^{*}\right)=\sigma_{S(\omega)}\left(z^{*}\right) .
$$

Since both sets are closed and convex, we can conclude that for all $\omega \in \Omega$, $S(\omega)=\operatorname{epi}(\omega, \cdot)$.

So we have shown that epi $f^{n}(\omega, \cdot) \stackrel{h}{\rightarrow}$ epi $(\omega, \cdot)$ for all $\omega \in \Omega$ as $n \rightarrow \infty$. Next, using the results of Hiai-Umegaki [8], for all $A \in \Sigma_{0}$, we can write

$$
\begin{array}{r}
\int_{A} h\left(E^{\Sigma_{0}} \text { epi } f^{n}(\omega, \cdot), E^{\Sigma_{0}} \text { epi } f^{m}(\omega, \cdot)\right) d \mu(\omega) \\
\leq \int_{A} h\left(\text { epi } f^{n}(\omega, \cdot), \text { epi } f^{m}(\omega, \cdot)\right) d \mu(\omega)
\end{array}
$$

and the latter tends to zero as $n, m \rightarrow \infty$. So for all $A \in \Sigma_{0}$, we have

$$
\lim _{n, m \rightarrow \infty} \int_{A} h\left(E^{\Sigma_{0}} \text { epi } f^{n}(\omega, \cdot), E^{\Sigma_{0}} \text { epi } f^{m}(\omega, \cdot)\right) d \mu(\omega) \rightarrow 0 .
$$

Using the Lebesgue dominated convergence theorem, we can pass the limit inside the integral and obtain

$$
\int_{A} \lim _{n, m \rightarrow \infty} h\left(E^{\Sigma_{0}} \text { epi } f^{n}(\omega, \cdot), E^{\Sigma_{0}} \text { epi } f^{m}(\omega, \cdot)\right) d \mu(\omega)=0 .
$$


Since this holds for all $A \in \Sigma_{0}$, we conclude that

$$
\lim _{n, m \rightarrow \infty} h\left(E^{\Sigma_{0}} \text { epi } f^{n}(\omega, \cdot), E^{\Sigma_{0}} \text { epi } f^{m}(\omega, \cdot)\right)=0 \quad \mu \text {-a.e. }
$$

Hence, $\left\{E^{\Sigma_{0}} \text { epi } f^{n}(\omega, \cdot)\right\}_{n \geq 1}$ is $\mu$-a.e. an $h$-Cauchy sequence in $P_{f c}(Z)$. Using once again the completeness of $\left(P_{f c}(Z), h\right)$, we get that there exists $G: \Omega \rightarrow P_{f c}(Z)$ s.t.

$$
E^{\Sigma_{0}} \text { epi } f^{n}(\omega, \cdot) \stackrel{h}{\rightarrow} G(\omega) \quad \mu \text {-a.e. }
$$

(we just set $G(\omega)=M \in P_{f c}(Z)$ on the exceptional $\mu$-null set). Note that $G(\cdot)$, being the limit in the Hausdorff metric of measurable multifunctions, is itself measurable. Also it is not difficult to check that

$$
\begin{aligned}
h\left(\operatorname{cl} \int_{A} E^{\Sigma_{0}} \text { epi } f^{n}(\omega, \cdot) d \mu(\omega), \operatorname{cl} \int_{A} G(\omega) d \mu(\omega)\right) \\
\leq \int_{A} h\left(E^{\Sigma_{0}} \text { epi } f^{n}(\omega, \cdot), G(\omega)\right) d \mu(\omega) \rightarrow 0 .
\end{aligned}
$$

So

$$
\operatorname{cl} \int_{A} E^{\Sigma_{0}} \text { epi } f^{n}(\omega, \cdot) d \mu(\omega) \rightarrow \operatorname{cl} \int_{A} G(\omega) d \mu(\omega) .
$$

But from [8] we know that

$$
\operatorname{cl} \int_{A} \text { epi } f^{n}(\omega, \cdot) d \mu(\omega)=\operatorname{cl} \int_{A} E^{\Sigma_{0}} \text { epi } f^{n}(\omega, \cdot) d \mu(\omega) .
$$

Taking $h$-limits as $n \rightarrow \infty$, we finally get

$$
\operatorname{cl} \int_{A} \operatorname{epi} f(\omega, \cdot) d \mu(\omega)=\operatorname{cl} \int_{A} G(\omega) d \mu(\omega)
$$

for all $A \in \Sigma_{0}$. Hence $E^{\Sigma_{0}}$ epi $\mathrm{f}(\omega, \cdot)=G(\omega)$.

Now if $(x, y) \in \operatorname{epi}(\omega, \cdot)$, then $f(\omega, x) \leq y$, and integrating we get

$$
\int_{A} f(\omega, x) d \mu(\omega)=\int_{A} g(\omega, x) d \mu(\omega) \leq \int_{A} y d \mu(\omega) .
$$

Since this is true for all $A \in \Sigma_{0}$, we get $g(\omega, x) \leq y$ so $(x, y) \in \operatorname{epi} g(\omega, \cdot)$. Hence epi $f(\omega, \cdot) \subseteq$ epi $g(\omega, \cdot)$ and, using the monotonicity of the set valued conditional expectation, we finally obtain

$$
E^{\Sigma_{0}} \text { epi } f(\omega, \cdot) \subseteq E^{\Sigma_{0}} \text { epi } g(\omega, \cdot)=\text { epi } g(\omega, \cdot) .
$$

Remarks. (1) Since $f(\cdot, \cdot)$ is bounded by $\phi(\cdot) \in L^{\infty}(\Omega)$, there will be an $n_{0}$ s.t. for all $n \geq n_{0}$, epi $f^{n}(\omega, \cdot) \neq \varnothing$. So the sequence 
$\left\{\text { epi } f^{n}(\omega, \cdot)\right\}_{n \geq 1}$ is well defined in $P_{f c}(Z)$ and without loss of generality we may assume that $n_{0}=1$.

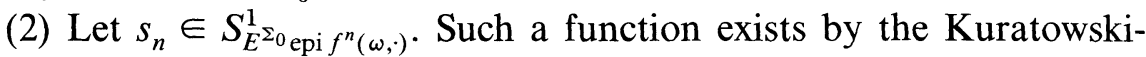
Ryll Nardzewski measurable selection theorem (see [9]). From the definition of the Hausdorff metric, we have

$$
\int_{A}\left\|s_{n}(\omega)-G(\omega)\right\| d \mu(\omega) \leq \int_{A} h\left(E^{\Sigma_{0}} \text { epi } f^{n}(\omega, \cdot), G(\omega)\right) d \mu(\omega) \rightarrow 0
$$

Also, since $Z$ is reflexive and $G(\omega)$ is convex, again by the same selection theorem, there is a measurable function $s: A \rightarrow Y$ s.t. $s(\omega) \in G(\omega)$ for all $\omega \in A$. Hence,

$$
S_{n} \stackrel{L_{Y}^{1}}{\rightarrow} S,
$$

so, for all $A \in \Sigma_{0}, \int_{A} G(\omega) d \mu(\omega) \neq \varnothing$.

In $\S 3$ we introduced the recession opetator $\left(f 0^{+}\right)(\cdot)$. Here, once again using the fact that, for $f: \Omega \times X \rightarrow Y$ a Caratheodory integrand operator s.t. $|f(\omega, x)| \leq g(\omega) \mu$-a.e. $g(\cdot) \in L_{Y}^{1}(\Omega)$, we have for all $u(\cdot), h(\cdot) \in$ $L_{X}^{1}(\Omega)$,

$$
\left(f 0^{+}\right)(\omega, h(\omega))=\mathrm{w}_{\lambda_{n} \rightarrow \infty} \frac{f\left(\omega, u(\omega)+\lambda_{n} h(\omega)\right)-f(\omega, u(\omega))}{\lambda_{n}},
$$

and we have the following result.

THEOREM 4.6. If $f: \Omega \times X \rightarrow Y$ is as above then for all $h(\cdot) \in L_{X}^{1}(\Omega)$,

$$
E^{\Sigma_{0}}\left(f 0^{+}\right)(\omega, h(\omega))=\left(E^{\Sigma_{0}} f\right) 0^{+}(\omega, h(\omega)) \quad \mu-a . e .,
$$

i.e. the operations of conditional expectation with respect to $\Sigma_{0}$ and of the recession operator commute.

We will close this section with a result on martingales formed by Caratheodory convex integrand operators. For that purpose consider an increasing sequence $\left\{\Sigma_{n}\right\}_{n \geq 1}$ of sub $\sigma$-fields of $\Sigma$. We set $\Sigma_{\infty}=\bigvee_{n=1}^{\infty} \Sigma_{n}$ and assume that $Y$ is reflexive, $X$ is finite dimensional and $h \in L^{\infty}$.

THEOREM 4.7. If $\left\{f_{n}, \Sigma_{n}\right\}_{n \geq 1}$ is a uniformly integrable martingale of Caratheodory convex integrand operators s.t. $\left\{f_{n}(\omega, \cdot)\right\}_{n \geq 1}$ is equicontinuous and $\sup _{n \geq 1} \int_{\Omega}\left\|f_{n}(\omega, x)\right\| d \mu(\omega)<\infty$ for all $x \in X$, then there exists 
$f_{\infty}: \Omega \times X \rightarrow Y$, also a Caratheodory convex integrand operator, s.t.

$$
f_{n}(\omega, x) \stackrel{\mathrm{s}}{\rightarrow} f_{\infty}(\omega, x) \quad \mu-a . e .
$$

and furthermore, $\left\{f_{n}, \Sigma_{n}\right\}_{n \in N \cup\{\infty\}}$ is a martingale too.

Proof. For any $x \in X, \sup _{n \geq 1} \int_{\Omega}\left\|f_{n}(\omega, x)\right\| d \mu(\omega)< \pm \infty$, we know from Chatterji's convergence theorem (see [5]) and the Ascoli Theorem that there exists $f_{\infty}: \Omega \times X \rightarrow Y$ s.t., for all $x \in X, f_{\infty}(\omega, x) \in L_{Y}^{1}(\Omega)$, and for which $f_{n}(\omega, x) \stackrel{s}{\rightarrow} f_{\infty}(\omega, x) \mu$-a.e. for all $x \in X$. Fix $\omega \in \Omega \backslash N_{0}$ where $\mu\left(N_{0}\right)=0$ and consider the operator $x \rightarrow f(\omega, x)$. We know that it is continuous. Also since for each $n \geq 1, f_{n}(\omega, \cdot)$ is a convex operator, then for $x_{1}, x_{2} \in X$ and $\lambda \in(0,1)$ we have

$$
f_{n}\left(\omega, \lambda x_{1}+(1-\lambda) x_{2}\right) \leq \lambda f_{n}\left(\omega, x_{1}\right)+(1-\lambda) f_{n}\left(\omega, x_{2}\right) .
$$

But recall that $f_{n}(\omega, x) \stackrel{s}{\rightarrow} f_{\infty}(\omega, x) \mu$-a.e. and $Y_{+}$is closed. So taking limits as $n \rightarrow \infty$, we get

$$
\begin{aligned}
& f_{\infty}\left(\omega, \lambda x_{1}+(1-\lambda) x_{2}\right) \\
& \leq \lambda f_{\infty}\left(\omega, x_{1}\right)+(1-\lambda) f_{\infty}\left(\omega, x_{2}\right) \quad \mu \text {-a.e., }
\end{aligned}
$$

which shows that $f_{\infty}(\omega, \cdot)$ is $\mu$-a.e. convex. Again by appropriately redefining the function on a $\mu$-null set, we have that $f(\omega, \cdot)$ is convex for all $\omega \in \Omega$. Furthermore, for $n>m$ and $A \in \Sigma_{m}$, we have

$$
\int_{A} f_{n}(\omega, x) d \mu(\omega)=\int_{A} f_{m}(\omega, x) d \mu(\omega)
$$

From the uniform integrability assumption, we know (see [5] or [6]) that

$$
f_{n} \stackrel{L_{Y}^{1}(\Omega)}{\rightarrow} f_{\infty}
$$

Hence

$$
\int_{A} f_{n}(\omega, x) d \mu(\omega) \rightarrow \int_{A} f_{\infty}(\omega, x) d \mu(\omega) \text { as } n \rightarrow \infty
$$

for all $A \in \Sigma_{m}$. From (*), keeping $m$ fixed and sending $n \rightarrow \infty$, we get

$$
\int_{A} f_{\infty}(\omega, x) d \mu(\omega)=\int_{A} f_{m}(\omega, x) d \mu(\omega) \text { for all } A \in \Sigma_{m},
$$


which means

$$
E^{\Sigma_{m}} f_{\infty}(\omega, x)=f_{m}(\omega, x) \mu \text {-a.e. for all } x .
$$

Hence $\left\{f_{n}, \Sigma_{n}\right\}_{n \in N \cup\{\infty\}}$ is a martingale.

REMARKS. (1) The uniform integrability was used only in proving that $\left\{f_{n}, \Sigma_{n}\right\}_{n \in N \cup\{\infty\}}$ is a martingale too. For the first part of the conclusion of the theorem, we only needed to know that $f_{n}(\omega, x) \stackrel{s}{\rightarrow} f_{\infty}(\omega, x) \mu$-a.e. for all $x \in X$. This is always the case when $Y$ has the Radon Nikodym property and Doob's condition $\sup _{n \geq 1}\left\|f_{n}\right\|_{L_{Y}^{1}(\Omega)}<+\infty$ is satisfied.

(2) We have an analogous result for submartingales of Caratheodory convex integrand operators by using the fact that a submartingale $\left\{f_{n}(\cdot, x)\right\}_{n \geq 1}$ can be written as $f_{n}(\omega, x)=m_{n}(\omega, x)+p_{n}(\omega, x)$, where $\left\{m_{n}(\cdot, x)\right\}_{n \geq 1}$ is a martingale and $\left\{p_{n}(\cdot, x)\right\}_{n \geq 1}$ a predictable sequence, i.e. $p_{n}(\cdot, x) \in L_{Y}^{1}\left(\Omega, \Sigma_{n-1}\right), p_{0}=0$ and $0 \leq p_{n}(\cdot, x) \uparrow \mu$-a.e. The decomposition is unique, and for $Y=R$ we recognize the well-known Doob decomposition of submartingales.

5. Nonconvex operators. In this section we prove two results where the operators involved are no longer convex, and we close our work with a vectorial generalization of Jensen's inequality.

We start with operators satisfying a general set-valued Lipschitz relation. So we have

THEOREM 5.1. If $Y^{*}$ is separable, $M: \Omega \rightarrow P_{f c}(Y)$ is an integrably bounded multifunction, and $f: \Omega \times X \rightarrow Y$ is an $L_{Y}^{1}(\Omega)$-integrand s.t. $f(\omega, x)-f(\omega, z) \in\|x-z\| M(\omega) \mu$-a.e. for all $x, z \in X$, then,

$$
E^{\Sigma_{0}} f(\omega, x)-E^{\Sigma_{0}} f(\omega, z) \in\|x-z\| E^{\Sigma_{0}} M(\omega) \quad \mu-a . e .
$$

Proof. First note that since, by hypothesis, $f(\cdot, x) \in L_{Y}^{1}(\Omega)$ for all $x \in X$, it has a vector valued conditional expectation with respect to $\Sigma_{0}$ and it belongs to $L_{Y}^{1}\left(\Omega, \Sigma_{0}\right)$ for all $x \in X$ (see [6]). So let $g(\omega, x)=$ $E^{\Sigma_{0}} f(\omega, x)$. Since, by hypothesis, we know that $f(\omega, x)-f(\omega, z) \in$ $\|x-z\| M(\omega) \mu$-a.e., for every $A \in \Sigma_{0}$ we have

$$
\int_{A}(f(\omega, x)-f(\omega, z)) d \mu(\omega) \in\|x-z\| \int_{A} M(\omega) d \mu(\omega) .
$$

But

$$
\int_{A}(f(\omega, x)-f(\omega, z)) d \mu(\omega)=\int_{A}(g(\omega, x)-g(\omega, z)) d \mu(\omega)
$$


and, since $M(\cdot)$ is a closed and convex valued integrable multifunction, we know that $E^{\Sigma_{0}} M(\cdot)$ exists as a closed and convex valued integrably bounded multifunction, and, furthermore, from Theorem 5.4 of [8], we know that

$$
\operatorname{cl} \int_{A} M(\omega) d \mu(\omega)=\operatorname{cl} \int_{A} E^{\Sigma_{0}} M(\omega) d \mu(\omega)
$$

Hence,

$$
\int_{A}(g(\omega, x)-g(\omega, z)) d \mu(\omega) \in\|x-z\| \mathrm{cl} \int_{A} E^{\Sigma_{0}} M(\omega) d \mu(\omega)
$$

for all $A \in \Sigma_{0}$. But then Lemma 4.4. of [8] tells us that

$$
g(\omega, x)-g(\omega, z) \in S_{\|x-z\| E^{\Sigma_{0 M}}}^{1},
$$

which finally implies that

$$
g(\omega, x)-g(\omega, z) \in\|x-z\| E^{\Sigma_{0}} M(\omega) \quad \mu \text {-a.e. }
$$

For the next result we consider the extended space $\hat{Y}=Y \cup\{ \pm \infty\}$. In [15] Penot introduced the following radial Dini like derivative for a general operator $f: X \rightarrow \bar{Y}$ :

$$
\underline{d}_{r} f(x ; h)=\frac{\lim }{\lambda \downarrow 0} \frac{f(x+\lambda h)-f(x)}{\lambda} .
$$

Along with it, he introduced a corresponding lower radial subdifferential as follows:

$$
\underline{\partial}_{r} f(x)=\left\{A \in \mathscr{L}(X, Y): A(h) \leq \underline{d}_{r} f(x ; h) \text { for all } h \in X\right\} .
$$

For a comparison of the subdifferential with the convex and the generalized (Clarke) subdifferential (as defined in [13]), we refer to [14].

In the next theorem we study the subdifferential of an $L_{Y}^{1}(\Omega)$-integrand for which the above directional derivative is finite. For more generality, let $Y$ be any normed lattice (not necessarily separable).

THEOREM 5.2. If $f: \Omega \times X \rightarrow Y$ is an $L_{Y}^{1}(\Omega)$-integrand s.t., for all $h \in X, \underline{d}_{r} f(\cdot, x ; h) \in L_{Y}^{1}(\Omega)$, then, for $F(x)=\int_{\Omega} f(\omega, x) d \mu(\omega)$,

$$
\underline{\partial}_{r} F(x) \supseteq \int_{\Omega} \underline{\partial}_{r} f(\omega, x) d \mu(\omega) .
$$


Proof. By definition

$$
\begin{aligned}
\underline{d}_{r} F(x ; h) & =\frac{\lim }{\lambda \downarrow 0} \frac{F(x+\lambda h)-F(x)}{\lambda} \\
& =\frac{\lim }{\lambda \downarrow 0} \int_{\Omega} \frac{f(\omega, x+\lambda h)-f(\omega, x)}{\lambda} d \mu(\omega) .
\end{aligned}
$$

We know that

$$
\begin{aligned}
& \frac{\lim }{\lambda \downarrow 0} \int_{\Omega} \frac{f(\omega, x+\lambda h)-f(\omega, x)}{\lambda} d \mu(\omega) \\
& \quad=\bigvee_{n \geq 1} \bigwedge_{\lambda \leq 1 / n} \int_{\Omega} \frac{f(\omega, x+\lambda h)-f(\omega, x)}{\lambda} d \mu(\omega) \\
& \quad \geq \bigvee_{n \geq 1} \int_{\Omega} \bigwedge_{\lambda \leq 1 / n} \frac{f(\omega, x+\lambda h)-f(\omega, x)}{\lambda} d \mu(\omega) .
\end{aligned}
$$

Let

$$
\underline{d}_{r} f^{n}(\omega, x ; h)=\bigwedge_{\lambda \leq 1 / n} \frac{f(\omega, x+\lambda h)-f(\omega, x)}{\lambda} .
$$

Note that

$$
\underline{d}_{r} f^{n}(\omega, x ; h) \uparrow \underline{d}_{r} f(\omega, x ; h) \text { as } n \rightarrow \infty .
$$

So, for every $y^{*} \in Y_{+}^{*}$,

$$
\left(y^{*}, \underline{d}_{r} f^{n}(\omega, x ; h)\right) \uparrow\left(y^{*}, \underline{d}_{r} f(\omega, x ; h)\right) \text { as } n \rightarrow \infty .
$$

Applying the monotone convergence theorem, we obtain

$$
\begin{array}{r}
\int_{\Omega}\left(y^{*}, \underline{d}_{r} f^{n}(\omega, x ; h)\right) d \mu(\omega) \uparrow \int_{\Omega}\left(y^{*}, \underline{d}_{r} f(\omega, x ; h)\right) d \mu(\omega) \\
\text { as } n \rightarrow \infty,
\end{array}
$$

so

$$
\begin{array}{r}
\left(y^{*}, \int_{\Omega} \underline{d}_{r} f^{n}(\omega, x ; h) d \mu(\omega)\right) \uparrow\left(y^{*}, \int_{\Omega} \underline{d}_{r} f(\omega, x ; h) d \mu(\omega)\right) \\
\text { as } n \rightarrow \infty .
\end{array}
$$

But we know from Krein's theorem (see [16]) that $Y_{+}^{*}$ is generating, i.e. $Y^{*}=Y_{+}^{*}-Y_{+}^{*}$. So, for all $y^{*} \in Y^{*}$,

$$
\left(y^{*}, \int_{\Omega} \underline{d}_{r} f^{n}(\omega, x ; h) d \mu(\omega)\right) \rightarrow\left(y^{*}, \int_{\Omega} \underline{d}_{r} f(\omega, x ; h) d \mu(\omega)\right),
$$


which means that

$$
\int_{\Omega} \underline{d}_{r} f^{n}(\omega, x ; h) d \mu(\omega) \stackrel{\mathrm{w}}{\rightarrow} \int_{\Omega} \underline{d}_{r} f(\omega, x ; h) d \mu(\omega) .
$$

However, we know from Schaeffer [23] that in a normed lattice every weakly convergent directed family is strongly convergent. So we obtain

$$
\int_{\Omega} \underline{d}_{r} f^{n}(\omega, x ; h) d \mu(\omega) \stackrel{\mathrm{s}}{\rightarrow} \int_{\Omega} \underline{d}_{r} f(\omega, x ; h) d \mu(\omega) .
$$

Since $\left\{\int_{\Omega} d_{r} f^{n}(\omega, x ; h) d \mu(\omega)\right\}_{n \geq 1}$ is increasing, using Lemma 5.8 of Schaeffer [23], we obtain

$$
\bigvee_{n \geq 1} \int_{\Omega} \underline{d}_{r} f_{\omega}^{n}(x ; h) d \mu(\omega)=\int_{\Omega} \underline{d}_{r} f(\omega, x ; h) d \mu(\omega) .
$$

Hence, we conclude that

$$
\underline{d}_{r} F(x ; h) \geq \int_{\Omega} \underline{d}_{r} f(\omega, x ; h) d \mu(\omega) .
$$

So let $T(\omega) \in \underline{\partial}_{r} f(\omega, x) \mu$-a.e. Then $T(\omega, h) \leq \underline{d}_{r} f(\omega, x ; h) \mu$-a.e. for all $h \in X$. Hence

$$
\int_{\Omega} T(\omega, h) d \mu(\omega) \leq \underline{d}_{r} F(x ; h) \quad \text { for all } h \in X,
$$

and if we set $T(h)=\int_{\Omega} T(\omega, h) d \mu(\omega)$, we have $T(h) \leq \underline{d}_{r} F(x ; h)$. Clearly $T \in \mathscr{L}(X, Y)$. So $T \in \underline{\partial} F(x)$. Therefore we conclude that

$$
\underline{\partial} F(x) \supseteq \int_{\Omega} \underline{\partial}_{r} f(\omega, x) d \mu(\omega) .
$$

We close with an interesting vectorial generalization of Jensen's inequality for both the unconditional and the conditional expectations.

THEOREM 5.3. If $u: X \rightarrow Y$ is a continuous convex operator s.t., for all $v(\cdot) \in L_{X}^{1}(\Omega),\|(u \circ v)(\omega)\| \leq h(\omega) \mu$-a.e., where

$$
h(\cdot) \in L^{1}(\Omega) \text { and } f(\cdot) \in L_{X}^{1}(\Omega) \text {, }
$$

then

$$
u\left[\int_{\Omega} f(\omega) d \mu(\omega)\right] \leq \int_{\Omega}(u \circ f)(\omega) d \mu(\omega) .
$$

Furthermore,

$$
u \circ E^{\Sigma_{0}} f(\omega) \leq E^{\Sigma_{0}}(u \circ f)(\omega) \quad \mu-a . e .
$$


Proof. Let $\left\{s_{n}(\cdot)\right\}_{n \geq 1}$ be simple functions s.t. $s_{n}(\omega) \stackrel{\text { s }}{\rightarrow} f(\omega) \mu$-a.e. and $\int_{\Omega} s_{n}(\omega) d \mu(\omega) \stackrel{\text { s }}{\rightarrow} \int_{\Omega} f(\omega) d \mu(\omega)$. Such a sequence exists from the definition of the Bochner integral. Let $s_{n}(\omega)=\sum_{k=1}^{m} \chi_{A_{k}}(\omega) w_{k}^{n}$.

$$
u\left[\int_{\Omega} s_{n}(\omega) d \mu(\omega)\right]=u\left[\int_{\Omega} \sum_{k=1}^{m} \chi_{A_{k}}(\omega) w_{k}^{n} d \mu(\omega)\right]=u\left[\sum_{k=1}^{m} \mu\left(A_{k}\right) w_{k}^{n}\right] .
$$

But observe that, for all $k \in\{1, \ldots, m\}, \mu\left(A_{k}\right) \in[0,1]$ and $\sum_{k=1}^{m} \mu\left(A_{k}\right)=$ $\mu(\Omega)=1$. So using the convexity of $u(\cdot)$, we get

$$
u\left[\int_{\Omega} s_{n}(\omega) d \mu(\omega)\right] \leq \sum_{k=1}^{m} \mu\left(A_{k}\right) u\left(w_{k}^{n}\right) .
$$

On the other hand, we have

$$
\text { (2) } \begin{aligned}
\int_{\Omega} u\left(s_{n}(\omega)\right) d \mu(\omega) & =\int_{\Omega} u\left(\sum_{k=1}^{m} \chi_{A_{k}}(\omega) w_{k}^{n}\right) d \mu(\omega) \\
& =\sum_{k=1}^{m} \int_{A_{k}} u\left(w_{k}^{n}\right) d \mu(\omega)=\sum_{k=1}^{m} \mu\left(A_{k}\right) u\left(w_{k}^{n}\right) .
\end{aligned}
$$

From (1) and (2), we conclude that

$$
u\left[\int_{\Omega} s_{n}(\omega) d \mu(\omega)\right] \leq \int_{\Omega} u\left(s_{n}(\omega)\right) d \mu(\omega) .
$$

But, since $s_{n}(\omega) \stackrel{s}{\rightarrow} f(\omega) \mu$-a.e. and $\int_{\Omega} s_{n}(\omega) d \mu(\omega) \stackrel{s}{\rightarrow} \int_{\Omega} f(\omega) d \mu(\omega)$, and because $u(\cdot)$ is, by hypothesis, continuous, we have

$$
u\left[\int_{\Omega} s_{n}(\omega) d \mu(\omega)\right] \stackrel{\mathrm{s}}{\rightarrow} u\left[\int_{\Omega} f(\omega) d \mu(\omega)\right]
$$

and

$$
\int_{\Omega} u\left(s_{n}(\omega)\right) d \mu(\omega) \stackrel{\mathrm{s}}{\rightarrow} \int_{\Omega} u(f(\omega)) d \mu(\omega),
$$

which proves the vectorial Jensen inequality for Bochner integrals. Next, since $E^{\Sigma_{0}}(\cdot)$ is a linear contraction on $L_{X}^{1}(\Omega)$ (see [6]), we have $E^{\Sigma_{0}} S_{n} \stackrel{L_{X}^{1}(\Omega)}{\rightarrow} E^{\Sigma_{0}} f$. By passing to a subsequence, if necessary, we may assume $E^{\Sigma_{0}} s_{n}(\omega) \stackrel{\mathrm{s}}{\rightarrow} E^{\Sigma_{0}} f(\omega) \mu$-a.e., and since, by hypothesis, $u(\cdot)$ is continuous, we have

$$
\left(u \circ E^{\Sigma_{0}} s_{n}\right)(\omega)=u \circ E^{\Sigma_{0}}\left(\sum_{k=1}^{m} \chi_{A_{k}} w_{k}^{n}\right)(\omega)=u \circ \sum_{k=1}^{m} w_{k}^{n} E^{\Sigma_{0}} \chi_{A_{k}}(\omega) .
$$


However, note that $0 \leq E^{\Sigma_{0}} \chi_{A_{k}}(\omega) \leq 1$ for $\omega \in \Omega$ and

$$
\sum_{k=1}^{m} E^{\Sigma_{0}} \chi_{A_{k}}(\omega)=E^{\Sigma_{0}} \chi_{\Omega}(\omega)=E^{\Sigma_{0}} 1=1
$$

Hence, using the fact that $u(\cdot)$ is convex, we have

$$
\left(u \circ E^{\Sigma_{0}} s_{n}\right)(\omega) \leq \sum_{k=1}^{m} E^{\Sigma_{0}} \chi_{A_{k}}(\omega) u\left(w_{k}^{n}\right) .
$$

Integrating, we get

$$
\int_{A}\left(u \circ E^{\Sigma_{0}} s_{n}\right)(\omega) d \mu(\omega) \leq \sum_{k=1}^{m} \mu\left(A \cap A_{k}\right) u\left(w_{k}^{n}\right) .
$$

Also

$$
\int_{A} E^{\Sigma_{0}}\left(u \circ \sum_{k=1}^{m} w_{k}^{n} \chi_{A_{k}}\right)(\omega)=\sum_{k=1}^{m} \mu\left(A \cap A_{k}\right) u\left(w_{k}^{n}\right) .
$$

From (4) and (5) we deduce that, for all $A \in \Sigma_{0}$,

$$
\left(u \circ E^{\Sigma_{0}} s_{n}\right)(\omega) \leq E^{\Sigma_{0}}\left(u \circ s_{n}\right)(\omega) \quad \mu \text {-a.e., }
$$

and passing to the limit we finally have

$$
\left(u \circ E^{\Sigma_{0}} f\right)(\omega) \leq E^{\Sigma_{0}}(u \circ f)(\omega) \quad \mu \text {-a.e.. }
$$

\section{REFERENCES}

[1] J. P. Aubin, Mathematical Methods of Game and Economic Theory, Studies in Math and Its Applications, Vol. 7, North Holland, Amsterdam (1977).

[2] R. Aumann, Integrals of set valued functions, J. Math. Anal. Appl., 12 (1965), 1-12.

[3] J. M. Bismut, Intégrales convexes et Probabilités, J. Math. Anal. Appl., 42 (1973), 639-73.

[4] C. Castaing-M. Valadier, Convex analysis and measurable multifunctions, Lecture Notes in Math Vol. 580 Springer, Berlin (1976).

[5] S. D. Chatterji, Martingale convergence and the Radon-Nikodym theorem in Banach Spaces, Math. Scand., 22 (1968), $21-41$.

[6] J. Diestel-J. J. Uhl, Jr., Vector Measures, Mathematical Surveys, Vol. 15, Amer. Math. Soc., Providence, Rhode Island (1977).

[7] P. Halmos, A Hilbert Space Problem Book, Van Nostrand-Reinhold, New York (1967).

[8] F. Hiai-F. Umegaki, Integrals, conditional expectations, and martingales of multivalued functions, J. Mult. Anal., 7 (1977), 149-82.

[9] C. Himmelberg, Measurable relations, Fund. Math., LXXXVIII (1975), 53-72.

[10] J. B. Hiriart-Urruty, About properties of the mean functional and continuous inf convolution in stochastic convex analysis, in: Optimization Techniques, Modeling and Optimization in the Service of Man, Ed. J. Cea, Springer, Lecture Notes in Computer Science, Berlin (1976), 763-89. 
[11] N. Neumann, On the Strassen disintegration theorem, Arch. Math., 29 (1977), 413-20.

[12] N. S. Papageorgiou, Nonsmooth analysis on partially ordered vector spaces: Part I. Convex Case, Pacific J. Math., 107 (1983), 403-458.

[13] _ Nonsmooth analysis on partially ordered vector spaces: Part II. Nonconvex case, Clarke's theory, Pacific J. Math., 109 (1983), 463-495.

[14] __ Nonsmooth Analysis on Partially Ordered Vector Spaces: Part III. The Subdifferential Theory, Presented at the 11th Symposium on Math Programming, (Bonn-August 1982).

[15] J. P. Penot, Calcul sous differentiel et optimisation, J. Funct. Anal., 27 (1978), 248-76.

[16] A. Peressini, Ordered Topological Vector Spaces, Harper and Row, New York (1967).

[17] R. T. Rockafellar, Integrals which are convex functionals, Pacific J. Math., 24 (1968), 525-38.

[18] _ Integrals which are convex functionals II, Pacific J. Math., 39 (1971), 439-69.

[19] Convex Integral Functionals and Duality, in Contributions to Nonlinear Analysis, ed. Zarontonelli, p. 215-36, Academic Press, New York (1971).

[20] _ Integral Functionals, Normal Integrands and Measurable Selections, in Nonlinear Operators and the Calculus of Variations, ed. J. Gossez et al., p. 157-207, Lecture Notes in Math, Vol. 543, Springer, Berlin (1976).

[21] _ Convex Analysis, Princeton Univ. Press, Princeton (1970).

[22] J. Saint-Pierre, Une extension du théorème de Strassen, C.R.A.S. t 279 (1974), 5-8.

[23] H. H. Schaefer, Banach Lattices and Positive Operators, Springer, New York (1974).

[24] M. Valadier, Sous différentiabilite de fonctions convexes à valeurs dans un espace vectoriel ordonné, Math. Scand., 30 (1972), 65-74.

Received March 14, 1983. Research supported in part by the Joint Services of Electronics, No. N00014-75-C-0648.

UNIVERSITY OF ILLINOIS

URBANA, IL 61801. 


\section{PACIFIC JOURNAL OF MATHEMATICS \\ EDITORS}

Donald BABBITT (Managing Editor)

University of California

Los Angeles, CA 90024

J. Dugundu

University of Southern Californa

Los Angeles, CA 90089-1113

R. FINN

Stanford University

Stanford, CA 94305

HermanN FLaSChKa

University of Arizona

Tucson, AZ 85721

\author{
C. C. MOORE \\ University of California \\ Berkeley, CA 94720 \\ ARTHur Ogus \\ University of California \\ Berkeley, CA 94720 \\ Hugo Rossi \\ University of Utah \\ Salt Lake City, UT 84112 \\ H. SAMELSON \\ Stanford University \\ Stanford, CA 94305
}

ASSOCIATE EDITORS
R. ARENS
E. F. BECKENBACH
B. H. NeUmanN
F. WOLF
K. YOSHIDA (1906-1982)

\section{SUPPORTING INSTITUTIONS}

UNIVERSITY OF ARIZONA

UNIVERSITY OF BRITISH COLUMBIA

UNIVERSITY OF OREGON

CALIFORNIA INSTITUTE OF TECHNOLOGY

UNIVERSITY OF CALIFORNIA

MONTANA STATE UNIVERSITY

UNIVERSITY OF SOUTHERN CALIFORNIA

UNIVERSITY OF NEVADA, RENO

STANFORD UNIVERSITY

UNIVERSITY OF HAWAII

NEW MEXICO STATE UNIVERSITY

UNIVERSITY OF TOKYO

UNIVERSITY OF UTAH

WASHINGTON STATE UNIVERSITY

OREGON STATE UNIVERSITY

UNIVERSITY OF WASHINGTON 


\section{Pacific Journal of Mathematics}

\section{Vol. 116, No. $1 \quad$ November, 1985}

K. Adachi, Le problème de Lévi pour les fibrés grassmanniens et les variétés

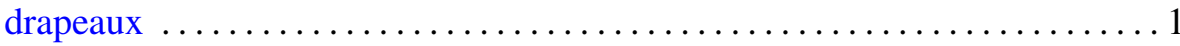

John MacLeod Ball, Remarks on the paper: "Basic calculus of variations" . . . 7 John Kelly Beem and Phillip E. Parker, Whitney stability of solvability . . . 11 Alberto Facchini, Decompositions of algebraically compact modules .......25

S. S. Khare, Finite group action and equivariant bordism $\ldots \ldots \ldots \ldots . \ldots 39$

Horst Leptin, A new kind of eigenfunction expansions on groups $\ldots \ldots \ldots . .45$

Pei-Kee Lin, Unconditional bases and fixed points of nonexpansive

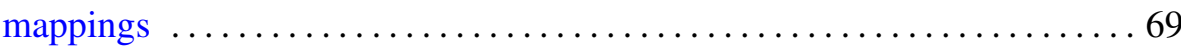

Charles Livingston, Stably irreducible surfaces in $S^{4} \ldots \ldots \ldots \ldots \ldots 77$

Kevin Mor McCrimmon, Nonassociative algebras with scalar involution . . .885

Albert Milani, Singular limits of quasilinear hyperbolic systems in a

bounded domain of $\mathbf{R}^{3}$ with applications to Maxwell's equations

Takemi Mizokami, On $M$-structures and strongly regularly stratifiable

spaces

Jesper M. Møller, On the homology of spaces of sections of complex

projective bundles

Nikolaos S. Papageorgiou, Carathéodory convex integrand operators and

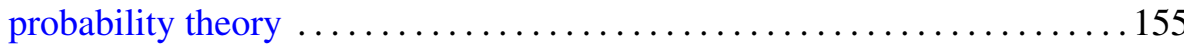

Robert John Piacenza, Transfer in generalized prestack cohomology 185

Lance W. Small and Adrian R. Wadsworth, Integrality of subrings of matrix rings ...

James Michael Wilson, On the atomic decomposition for Hardy spaces 